\title{
On the annual modulation signal in dark matter direct detection
}

\author{
Juan Herrero-Garcia, ${ }^{1,2, *}$ Thomas Schwetz, ${ }^{1, \dagger}$ and Jure Zupan ${ }^{\ddagger 3, \S}$ \\ ${ }^{1}$ Max-Planck-Institut für Kernphysik, PO Box 103980, 69029 Heidelberg, Germany \\ ${ }^{2}$ Departamento de Fisica Teorica, and IFIC, Universidad de Valencia-CSIC \\ Edificio de Institutos de Paterna, Apt. 22085, 46071 Valencia, Spain \\ ${ }^{3}$ Department of Physics, University of Cincinnati, Cincinnati, Ohio 45221,USA
}

\begin{abstract}
We derive constraints on the annual modulation signal in Dark Matter (DM) direct detection experiments in terms of the unmodulated event rate. A general bound independent of the details of the DM distribution follows from the assumption that the motion of the earth around the sun is the only source of time variation. The bound is valid for a very general class of particle physics models and also holds in the presence of an unknown unmodulated background. More stringent bounds are obtained, if modest assumptions on symmetry properties of the DM halo are adopted. We illustrate the bounds by applying them to the annual modulation signals reported by the DAMA and CoGeNT experiments in the framework of spin-independent elastic scattering. While the DAMA signal satisfies our bounds, severe restrictions on the DM mass can be set for CoGeNT.
\end{abstract}

\footnotetext{
$¥$ On leave of absence from University of Ljubljana, Depart. of Mathematics and Physics, Jadranska 19, 1000 Ljubljana, Slovenia and Josef Stefan Institute, Jamova 39, 1000 Ljubljana, Slovenia.

*Electronic address: juan.a.herrero AT uv.es

${ }^{\dagger}$ Electronic address: schwetz AT mpi-hd.mpg.de

$\S$ Electronic address: jure.zupan AT cern.ch
} 


\section{INTRODUCTION}

A smoking-gun signature of the dark matter (DM) signal in DM direct detection experiments is the annual modulation of the event rate. This arises because the earth rotates around the sun, while at the same time the sun moves relative to the DM halo [1, 2]. Currently, two experiments report annual modulation of the signals, DAMA with $8.9 \sigma$ significance [3], and CoGeNT with a significance of $2.8 \sigma$ [4]. An important question is whether the observed modulation rates are consistent with the interpretation that the signal is due to DM. Ideally, one would like to answer this question without requiring a detailed knowledge of the DM halo. As we will show below, we come very close to this ideal.

The DM scattering rate in the detector is determined by the particle physics properties of DM and by the astrophysical properties of the DM halo. The latter carry large uncertainties. When presenting results of DM direct detection experiments, it has become customary to use a "standard" Maxwellian DM velocity distribution in order to show the constraints on DM mass and scattering cross section. This is very likely an oversimplification, with N-body simulations indicating a more complicated structure of the DM halo, see e.g., [5]. It is well known that the annual modulation signal is sensitive to such deviations from the Maxwellian halo [6-8]. DM streams are particularly relevant for the annual modulation signal and can lead to large effects. These can, for instance, be comparable in size to the modulation effect due to the whole Maxwellian halo [9-12].

In the following we will derive constraints on the amplitude of the annual modulation using measured unmodulated rates. The derivation of these bounds is made possible by the fact that the velocity of the earth rotating around the sun, $v_{e} \sim 30 \mathrm{~km} / \mathrm{s}$, is much smaller than the typical velocity of DM particles in the halo, $\langle v\rangle \sim 200 \mathrm{~km} / \mathrm{s}$, and we can expand the velocity integral to first order in $v_{e}$. The derived constraints on the annual modulation then serve as a consistency check for the hypothesis that the annual modulation is due to the DM signal. The check that we provide is (almost) independent of DM halo properties. Previously halo independent methods to interpret DM direct detection data have been developed and applied in [13-18], with different goals than ours. In ref. [14] a method for determining the DM mass independently of the halo was developed, while refs. [13, 18] focused on extracting halo properties from the data. Compatibility studies of different experiments without adopting assumptions on the halo have been performed in [15-17]. Our goal is to obtain a consistency check between the modulation amplitude and the unmodulated signal within a given experiment.

The structure of the paper is as follows: in sec. 2 we set up the notation, followed in sec. 3 by a derivation of the general bound on the modulation signal under the very mild assumption that the properties of the DM halo in our vicinity do not change on time scales of months. In sec. 4 we impose further symmetry requirements on the DM velocity distribution and obtain successively more stringent bounds. In sec. 5 we then demonstrate the usefulness of our bounds by applying them to the modulation signals reported in CoGeNT and DAMA within elastic spin-independent scattering. We find that our method leads to non-trivial restrictions on DM interpretations of the modulation signal in CoGeNT whereas the DAMA signal satisfies our bounds for the relevant DM masses. We summarize in sec. 6 . In appendix A we consider the Maxwellian DM halo and show that the expansion in $v_{e}$ is rather accurate in this case. Furthermore, we illustrate the various bounds compared to the 
modulation signal expected for the Maxwellian halo. Appendix B provides technical details necessary to translate the bounds derived for the halo velocity integral into observable event rates. Supplementary material related to the treatment of an unknown background is given in appendix $\mathrm{C}$.

\section{NOTATION}

The differential rate in events $/ \mathrm{keV} / \mathrm{kg} /$ day for $\mathrm{DM} \chi$ to scatter off a nucleus $(A, Z)$ and depositing the nuclear recoil energy $E_{n r}$ in the detector is

$$
R\left(E_{n r}, t\right)=\frac{\rho_{\chi}}{m_{\chi}} \frac{1}{m_{A}} \int_{v>v_{m}} d^{3} v \frac{d \sigma_{A}}{d E_{n r}} v f_{\operatorname{det}}(\mathbf{v}, t) .
$$

Here $\rho_{\chi} \simeq 0.3 \mathrm{GeV} / \mathrm{cm}^{3}$ is the local DM density, $m_{A}$ and $m_{\chi}$ are the nucleus and DM masses, $\sigma_{A}$ the DM-nucleus scattering cross section $^{1}$ and $\mathbf{v}$ the 3 -vector relative velocity between $\mathrm{DM}$ and the nucleus, while $v \equiv|\mathbf{v}|$. For a DM particle to deposit recoil energy $E_{n r}$ in the detector a minimal velocity $v_{m}$ is required, restricting the integral over velocities in (1). For elastic scattering it is

$$
v_{m}=\sqrt{\frac{m_{A} E_{n r}}{2 \mu_{\chi A}^{2}}},
$$

where $\mu_{\chi A}$ is the reduced mass of the DM-nucleus system.

The function $f_{\text {det }}(\mathbf{v}, t)$ describes the distribution of DM particle velocities in the detector rest frame with $f_{\text {det }}(\mathbf{v}, t) \geq 0$ and $\int d^{3} v f_{\text {det }}(\mathbf{v}, t)=1$. It is related to the velocity distribution in the rest frame of the sun by

$$
f_{\text {det }}(\mathbf{v}, t)=f_{\text {sun }}\left(\mathbf{v}+\mathbf{v}_{e}(t)\right),
$$

where $\mathbf{v}_{e}(t)$ is the velocity vector of the earth, which we write as [9]

$$
\mathbf{v}_{e}(t)=v_{e}\left[\mathbf{e}_{1} \sin \lambda(t)-\mathbf{e}_{2} \cos \lambda(t)\right]
$$

with $v_{e}=29.8 \mathrm{~km} / \mathrm{s}$, and $\lambda(t)=2 \pi(t-0.218)$ with $t$ in units of 1 year and $t=0$ at January 1st, while $\mathbf{e}_{1}=(-0.0670,0.4927,-0.8676)$ and $\mathbf{e}_{2}=(-0.9931,-0.1170,0.01032)$ are orthogonal unit vectors spanning the plane of the earth's orbit, assumed to be circular. Similarly, the DM velocity distribution in the galactic frame is connected to the one in the rest frame of the sun by $f_{\text {sun }}(\mathbf{v})=f_{\text {gal }}\left(\mathbf{v}+\mathbf{v}_{\text {sun }}\right)$, with $\mathbf{v}_{\text {sun }} \approx(0,220,0) \mathrm{km} / \mathrm{s}+\mathbf{v}_{\text {pec }}$ and $\mathbf{v}_{\text {pec }} \approx(10,13,7) \mathrm{km} / \mathrm{s}$ the peculiar velocity of the sun. We are using galactic coordinates where $x$ points towards the galactic center, $y$ in the direction of the galactic rotation, and $z$ towards the galactic north, perpendicular to the disc. As shown in [7], eq. (4) provides an excellent approximation to describe the annual modulation signal.

\footnotetext{
${ }^{1}$ Throughout this work we assume that DM is dominated by a single particle species. A generalization to
} multi-component DM is straightforward and will be pursued in future work. 
In the following we consider the typical situation, where the differential cross section is given by

$$
\frac{d \sigma_{A}}{d E_{n r}}=\frac{m_{A}}{2 \mu_{\chi A}^{2} v^{2}} \sigma_{A}^{0} F^{2}\left(E_{n r}\right)
$$

where $\sigma_{A}^{0}$ is the total DM-nucleus scattering cross section at zero momentum transfer, and $F\left(E_{n r}\right)$ is a form factor. The event rate is then given by

$$
R\left(E_{n r}, t\right)=C F^{2}\left(E_{n r}\right) \eta\left(v_{m}, t\right) \quad \text { with } \quad C=\frac{\rho_{\chi} \sigma_{A}^{0}}{2 m_{\chi} \mu_{\chi A}^{2}}
$$

and the halo integral

$$
\eta\left(v_{m}, t\right) \equiv \int_{v>v_{m}} d^{3} v \frac{f_{\mathrm{det}}(\mathbf{v}, t)}{v} .
$$

Here and in the following $v_{m}$ and $E_{n r}$ are related by eq. (2). This formalism covers a wide range of possible DM-nucleus interaction models, including the standard spin-independent and spin-dependent scattering. The results we derive below apply to all the cases where $d \sigma_{A} / d E_{n r} \propto 1 / v^{2}$, in which case the halo integral (7) is obtained. The arguments can easily be generalized to a non-trivial $q^{2}$ dependence of the interaction, which would introduce an additional $E_{n r}$ dependent function in (6) but would not change (7). Our results do not apply, however, to a non-standard velocity dependence of the cross section, which would modify eq. (7).

The rate will have a time independent component and an annually modulated component which we define as

$$
R\left(E_{n r}, t\right)=\bar{R}\left(E_{n r}\right)+\delta R\left(E_{n r}, t\right)=C F^{2}\left(E_{n r}\right)\left[\bar{\eta}\left(v_{m}\right)+\delta \eta\left(v_{m}, t\right)\right] .
$$

Below we will be specifically interested in purely sinusoidal time dependence with period of one year, in which case we can write

$$
\begin{aligned}
\delta R\left(E_{n r}, t\right) & =A_{R}\left(E_{n r}\right) \cos 2 \pi\left[t-t_{0}\left(E_{n r}\right)\right] \\
\delta \eta\left(v_{m}, t\right) & =A_{\eta}\left(v_{m}\right) \cos 2 \pi\left[t-t_{0}\left(E_{n r}\right)\right] .
\end{aligned}
$$

The peak of the annual modulation occurs at $t_{0}$, which in general depends on $E_{n r}$ (or equivalently on $v_{m}$ ). The modulation amplitudes of the event rate, $A_{R}$, and of the halo integral, $A_{\eta}$, are related through $A_{R}\left(E_{n r}\right)=C F^{2}\left(E_{n r}\right) A_{\eta}\left(v_{m}\right) \geq 0$. We will first derive bounds on $A_{\eta}$ in terms of the time averaged value of the halo integral $\bar{\eta}$. In section 5 we will then translate the bounds into constraints involving the observable quantities $A_{R}$ and $\bar{R}$.

\section{GENERAL BOUND ON THE ANNUAL MODULATION AMPLITUDE}

Assumption 1: We assume that the only time dependence comes from $\mathbf{v}_{e}(t)$ and there is no explicit time dependence in $f_{\text {sun }}$.

This assumption implies that the halo is spatially constant at the scale of the sun-earth distance and also constant in time on scale of months. Under this assumption we can derive 
a general bound on the annual modulation by expanding eq. (7) in $v_{e} / v_{m} \ll 1^{2}$. Using for short $f \equiv f_{\text {sun }}$, we have

$$
\begin{aligned}
\eta\left(v_{m}, t\right) & =\int_{\left|\mathbf{v}-\mathbf{v}_{e}\right|>v_{m}} d^{3} v \frac{f(\mathbf{v})}{\left|\mathbf{v}-\mathbf{v}_{e}\right|} \\
= & \int_{v>v_{m}} d^{3} v \frac{f(\mathbf{v})}{v}+\int d^{3} v f(\mathbf{v}) \frac{\mathbf{v} \cdot \mathbf{v}_{e}(t)}{v^{3}}\left[\Theta\left(v-v_{m}\right)-\delta\left(v-v_{m}\right) v_{m}\right]+\mathcal{O}\left(v_{e}^{2} / v_{m}^{2}\right)
\end{aligned}
$$

where the first term in (11) gives the time independent part of the DM scattering signal. In polar coordinates the time independent halo integral is then given by

$$
\bar{\eta}\left(v_{m}\right)=\int_{v>v_{m}} d^{3} v \frac{f(\mathbf{v})}{v}=\int_{v_{m}} d v v \int_{0}^{2 \pi} d \varphi \int_{-1}^{1} d \cos \vartheta f(v, \vartheta, \varphi) .
$$

The second term in (11) corresponds to the time dependent part of the DM scattering signal, with $\mathbf{v}_{e}(t)$ given in (4). Expanding to linear order in $v_{e}$ thus leads to an annual modulation signal that has a sinusoidal shape. One can check experimentally for the convergence of the expansion by searching for higher order terms, $\propto v_{e}^{2} \sin ^{2}\left[2 \pi\left(t-t_{0}\right)\right]$, etc. Note that the expansion is in $v_{e} / v$, where $v \gtrsim v_{m}$, so that the accuracy is typically better than $\mathcal{O}\left(v_{e} / v_{m}\right)$. In appendix $\mathrm{A}$ we demonstrate explicitly that this expansion is rather accurate in the case of a Maxwellian halo.

By expanding the velocity integral in the small quantity $v_{e}$ we assume that $f(\mathbf{v})$ is smooth on scales $\lesssim v_{e}$. Hence, our bounds do not apply in situations where $f(\mathbf{v})$ has strong structures at scales smaller than $v_{e}$. An example would be a very cold stream with velocity $v_{\text {stream }}$ and a dispersion smaller than $v_{e}$. In such a case the expansion will not be accurate for $v_{m}$ in the range $\left|v_{m}-v_{\text {stream }}\right| \lesssim v_{e} \sin \alpha$, though it may still work to good accuracy for $v_{m}$ outside this range.

The time dependent component in (11) has two contributions

$$
\delta \eta\left(v_{m}, t\right)=\int d^{3} v f(\mathbf{v}) \frac{\mathbf{v} \cdot \mathbf{v}_{e}(t)}{v^{3}}\left[\Theta\left(v-v_{m}\right)-\delta\left(v-v_{m}\right) v_{m}\right]
$$

The term with the $\Theta$-function comes from expanding the denominator in eq. (10) and involves a volume integral over the region $v>v_{m}$. The term with the $\delta$-function comes from taking into account the effect of $v_{e}$ on the integration boundary, and the $\delta$-function indicates that the argument has to be evaluated at the surface $v=v_{m}$. Let us treat the two terms separately and define

$$
\begin{aligned}
\hat{\mathbf{v}}_{g}\left(v_{m}\right) g\left(v_{m}\right) & \equiv \int d^{3} v f(\mathbf{v}) \frac{\mathbf{v}}{v^{3}} \delta\left(v-v_{m}\right) \\
\hat{\mathbf{v}}_{G}\left(v_{m}\right) G\left(v_{m}\right) & \equiv \int d^{3} v f(\mathbf{v}) \frac{\mathbf{v}}{v^{3}} \Theta\left(v-v_{m}\right) .
\end{aligned}
$$

\footnotetext{
2 Typically $v_{m}$ is sensitive to both the nucleus and the DM mass. For a $10 \mathrm{GeV}$ DM mass and a recoil energy $E_{n r}$ of a few $\mathrm{KeV}$, we get for the nuclei we have analysed (Ge, Na, I) that $v_{m} \gtrsim 5 v_{e} \mathrm{~km} / \mathrm{s}$, so the expansion is rather accurate (as we have checked explicitly for a Maxwellian halo in appendix A).
} 
The unit vectors $\hat{\mathbf{v}}_{g}\left(v_{m}\right)$ and $\hat{\mathbf{v}}_{G}\left(v_{m}\right)$ give the corresponding weight averaged DM wind directions in the earth's rest frame. In general they point in different directions and can depend on $v_{m}$. For the Maxwellian halo they point in the same direction and are equal to $\hat{\mathbf{v}}_{g}=\hat{\mathbf{v}}_{G}=-\mathbf{v}_{\text {sun }}$. We will treat such special cases in the next section. The positive functions $g\left(v_{m}\right)$ and $G\left(v_{m}\right)$ are given by

$$
\begin{aligned}
g\left(v_{m}\right) & =\int_{0}^{2 \pi} d \varphi \int_{-1}^{1} d \cos \vartheta f\left(v_{m}, \vartheta, \varphi\right) \cos \vartheta \\
G\left(v_{m}\right) & =\int_{v_{m}} d v \int_{0}^{2 \pi} d \varphi \int_{-1}^{1} d \cos \vartheta^{\prime} f\left(v, \vartheta^{\prime}, \varphi\right) \cos \vartheta^{\prime}
\end{aligned}
$$

where $\vartheta\left(\vartheta^{\prime}\right)$ is the angle between $\mathbf{v}$ and $\hat{\mathbf{v}}_{g}\left(\hat{\mathbf{v}}_{G}\right)$. The time dependent halo integral is thus given by

$$
\delta \eta\left(v_{m}, t\right)=\mathbf{v}_{e}(t) \cdot\left[\hat{\mathbf{v}}_{G}\left(v_{m}\right) G\left(v_{m}\right)-\hat{\mathbf{v}}_{g}\left(v_{m}\right) v_{m} g\left(v_{m}\right)\right] .
$$

As already mentioned, the form of $\mathbf{v}_{e}(t)$ from eq. (4) implies that our approximations lead to strictly sinusoidal modulations, justifying the ansatz in eq. (9), $\delta \eta\left(v_{m}, t\right)=A_{\eta}\left(v_{m}\right) \cos 2 \pi(t-$ $\left.t_{0}\right)$. Using eq. (18), the modulation amplitude can be constrained in the following way:

$$
A_{\eta}\left(v_{m}\right) \leq v_{e}\left[v_{m} g\left(v_{m}\right)+G\left(v_{m}\right)\right] .
$$

Note that the two terms in eq. (18) will contribute to the modulation amplitude proportional to $\sin \alpha_{g}$ and $\sin \alpha_{G}$, respectively, where $\alpha_{g, G}$ is the angle between $\hat{\mathbf{v}}_{g, G}$ and the direction orthogonal to the plane of the earth orbit, i.e., $\cos \alpha_{g, G}=\hat{\mathbf{v}}_{g, G} \cdot \mathbf{e}_{\mathbf{3}}$, with $\mathbf{e}_{\mathbf{3}}=\mathbf{e}_{\mathbf{1}} \times \mathbf{e}_{\mathbf{2}}$, and $\mathbf{e}_{1,2}$ given below eq. (4). In general $\alpha_{g, G}$ will depend on $v_{m}$. To derive the bound we have assumed the maximal possible effect, corresponding to $\sin \alpha_{g}=\sin \alpha_{G}=1$. Since we do not know the relative sign of the contributions from $g\left(v_{m}\right)$ and $G\left(v_{m}\right)$ we have to take the sum of the moduli.

The function $g\left(v_{m}\right)$ is bounded from above by

$$
g\left(v_{m}\right) \leq \int d \varphi d \cos \vartheta f\left(v_{m}, \varphi, \vartheta\right)|\cos \vartheta| \leq \int d \varphi d \cos \vartheta f\left(v_{m}, \varphi, \vartheta\right)=-\frac{1}{v_{m}} \frac{d \bar{\eta}}{d v_{m}}
$$

where the last equality follows from eq. (12). Note that $\bar{\eta}\left(v_{m}\right)$ is a monotonously decreasing function and therefore, $d \bar{\eta}\left(v_{m}\right) / d v_{m} \leq 0$. Using again the last equality above, also a bound for $G\left(v_{m}\right)$ can be derived:

$$
G\left(v_{m}\right) \leq-\int_{v_{m}} d v \frac{1}{v} \frac{d \bar{\eta}}{d v}=\frac{\bar{\eta}\left(v_{m}\right)}{v_{m}}-\int_{v_{m}} d v \frac{\bar{\eta}(v)}{v^{2}} .
$$

The equality in (21) follows from integration by parts. The inequalities (20) and (21) are saturated if $f(\mathbf{v}) \propto \delta(\vartheta)$, i.e., the hypothetical situation that all DM particles have the same direction and their velocities have no transversal component. Using eqs. (20) and (21) the bound on the modulation amplitude (19) becomes

$$
A_{\eta}\left(v_{m}\right) \leq v_{e}\left[-\frac{d \bar{\eta}}{d v_{m}}+\frac{\bar{\eta}\left(v_{m}\right)}{v_{m}}-\int_{v_{m}} d v \frac{\bar{\eta}(v)}{v^{2}}\right] \quad \text { (Assumption 1) }
$$


where the first two terms on the r.h.s. are positive and the third is negative. If the DM scattering rate $\bar{R}$ is measured, the r.h.s. is fully determined experimentally by $\bar{\eta}\left(v_{m}\right)=\bar{R}\left(E_{n r}\right) / C F^{2}\left(E_{n r}\right)$, and can be compared to the observed modulation through $A_{\eta}=A_{R} / C F^{2}\left(E_{n r}\right)$, see section 5 for details. Note that the phase of the modulation (which may vary with $v_{m}$ ) does not appear in the bound (22). The bound applies on the modulation amplitude, irrespective of the phase. Eq. (22) is one of the main results of this paper. The bound is rather general and holds under the very mild Assumption 1 specified above. For it to be saturated the DM velocity distribution at the position of the solar system would need to be highly nontrivial. For instance, the bound in (19) can be saturated if there is a DM stream aligned with the ecliptic and it is strong enough so that it dominates the velocity distribution at $v=v_{m}$. Note that even in this case, for different $v_{m}$ the bound will not be saturated. To saturate in addition eq. (20) or (21), the stream should have no transversal velocity dispersion. Any modulation signal which violates, or even just saturates, this bound is very unlikely to have a DM origin.

\section{BOUNDS ON THE ANNUAL MODULATION FOR SYMMETRIC HALOS}

Assumption 2: In addition to Assumption 1 we now assume that $\hat{\mathbf{v}}_{g}$ defined in eq. (14) is independent of $v_{m}$, i.e., we assume that there is a constant direction $\hat{\mathbf{v}}_{\text {halo }} \equiv \hat{\mathbf{v}}_{g}$ governing the shape of the DM velocity distribution in the sun's rest frame.

From this assumption it follows immediately that $\hat{\mathbf{v}}_{G}$ is also constant and equal to $\hat{\mathbf{v}}_{\text {halo }}$, so that

$$
g\left(v_{m}\right)=-\frac{d G}{d v_{m}}
$$

and eq. (18) becomes

$$
\delta \eta\left(v_{m}, t\right)=-\mathbf{v}_{e}(t) \cdot \hat{\mathbf{v}}_{\text {halo }}\left[v_{m} g\left(v_{m}\right)-G\left(v_{m}\right)\right]
$$

The crucial difference to the general case (18) is that we were able to pull the velocity vector in front of the bracket. The functions $v_{m} g\left(v_{m}\right)$ and $G\left(v_{m}\right)$ are both positive. Their relative sizes determine whether the bracket is positive or negative. For small $v_{m}$ the function $G\left(v_{m}\right)$ typically dominates and we get an extra half a year shift in the peak of the modulation (for the Maxwellian halo the peak would then be in December). For larger $v_{m}$ the boundary term $v_{m} g\left(v_{m}\right)$ dominates and the whole bracket is positive (and thus for the Maxwellian halo the peak in this case is in June, see appendix A).

Assumption 2 is fulfilled if $f(\mathbf{v})$ obeys certain symmetry requirements that we can deduce from eq. (14). For a given $v_{m}$ we chose a coordinate system such that $\hat{\mathbf{v}}_{g}=(1,0,0)$, and

$$
\int d^{3} v f(\mathbf{v}) v_{y} \delta\left(v-v_{m}\right)=0, \quad \int d^{3} v f(\mathbf{v}) v_{z} \delta\left(v-v_{m}\right)=0
$$

Assumption 2 implies that these relations hold for any $v_{m}$ in the same coordinate system. The distribution in $v_{x}$ (i.e., in the direction of $\hat{\mathbf{v}}_{\text {halo }}$ ) can be arbitrary, and can for instance even include several peaks, as long as $\hat{\mathbf{v}}_{g}$ does not flip sign. Therefore, we have to require 
that the integral over the half-sphere with $v_{x}>0$ is larger than the one with $v_{x}<0$ for all $v_{m}$ :

$$
\int_{v_{x}<0} d^{3} v f(\mathbf{v}) v_{x} \delta\left(v-v_{m}\right)<\int_{v_{x}>0} d^{3} v f(\mathbf{v})\left|v_{x}\right| \delta\left(v-v_{m}\right) .
$$

One possibility to satisfy the condition (25) is a symmetric velocity distribution, with $f\left(v_{x}, v_{y}, v_{z}\right)=f\left(v_{x},-v_{y}, v_{z}\right)$ and $f\left(v_{x}, v_{y}, v_{z}\right)=f\left(v_{x}, v_{y},-v_{z}\right)$ for all $v_{x}$. Eq. (25) can also be satisfied for distributions asymmetric in $v_{y}$ and/or $v_{z}$, however, in this case the asymmetry has to be such that the cancellation between $v_{y, z}>0$ and $<0$ happens for all radii $v_{m}$.

Assumption 2 is fulfilled for the standard Maxwellian halo, as well as for any other isotropic velocity distribution. Up to small corrections due to the peculiar velocity of the sun it holds also for tri-axial halos, and covers also streams parallel to the motion of the sun, such as a dark disc co-rotating with the galactic stellar disc [19]. Note that for all those examples the DM direction $\hat{\mathbf{v}}_{\text {halo }}$ is aligned with the motion of the sun (up to the peculiar velocity that leads to sub-leading corrections). Let us introduce this as an additional assumption:

Assumption 2a: In addition to Assumption 2 we require that the preferred direction $\hat{\mathbf{v}}_{\text {halo }}$ is aligned with the motion of the sun relative to the galaxy.

As mentioned above, for many realistic cases fulfilling Assumption 2 also this additional requirement is fulfilled. An exception would be the situation when the DM density at the sun's location is dominated by a single stream from an arbitrary direction and the contribution of the static halo is negligible.

Let us now use eq. (24) to derive a bound on the modulation. We have $\mathbf{v}_{e}(t) \cdot \hat{\mathbf{v}}_{\text {halo }}=$ $-v_{e} \sin \alpha_{\text {halo }} \cos \left(t-t_{0}\right)$, where $t_{0}$ is now independent of $v_{m}$. Here $\alpha_{\text {halo }}$ is the angle between $\hat{\mathbf{v}}_{\text {halo }}$ and $\mathbf{e}_{1} \times \mathbf{e}_{2}$, i.e., the projection of $\hat{\mathbf{v}}_{\text {halo }}$ on the ecliptic plane, with $\sin \alpha_{\text {halo }} \geq 0$. Assumptions 2 and $2 \mathrm{a}$ thus imply that the phase of the modulation is independent of $v_{m}$ (and therefore independent of $E_{n r}$ ). As discussed above this is up to a sign flip of the square bracket in eq. (24) that can happen due to the two competing terms. To take this into account we now define

$$
\delta \eta\left(v_{m}, t\right)=A_{\eta}^{\prime}\left(v_{m}\right) \cos \left(t-t_{0}\right),
$$

where $t_{0}$ is constant and we allow a sign change for $A_{\eta}^{\prime}\left(v_{m}\right)$. This is different from $A_{\eta}$, which has been defined to be positive in eq. (9). While for Assumption 2 the phase is arbitrary but constant, Assumption 2a also gives a prediction for the phase - that the maximum (or minimum) of the event rate is around June 2nd. This can be checked in the experiment by looking at the annual modulation phase at different energy bins. Hence, from the experimental information on the phase we can conclude whether Assumptions 2 or 2a may be justified and whether it makes sense to apply the corresponding test.

A useful bound on the modulation can be obtained by first integrating eq. (24) over $v_{m}$,

$$
\begin{aligned}
\int_{v_{m 1}}^{v_{m 2}} d v_{m} A_{\eta}^{\prime}\left(v_{m}\right) & =v_{e} \sin \alpha_{\text {halo }} \int_{v_{m 1}}^{v_{m 2}} d v_{m}\left[v_{m} g\left(v_{m}\right)-G\left(v_{m}\right)\right] \\
& =v_{e} \sin \alpha_{\text {halo }}\left[v_{m 1} G\left(v_{m 1}\right)-v_{m 2} G\left(v_{m 2}\right)\right]
\end{aligned}
$$

where the second equality is obtained by integrating $v_{m} g\left(v_{m}\right)$ by parts using $g\left(v_{m}\right)=$ $-d G / d v_{m}$, eq. (23). Note that the $A_{\eta}^{\prime}$ and $\alpha_{\text {halo }}$ are defined in such a way that $A_{\eta}^{\prime}$ is 
positive for large $v_{m}$, above the last sign flip. Experimentally, this is at present the most interesting region of the parameter space. Both putative modulation signals, at CoGeNT and DAMA, have a peak close to June, and no half year phase change is seen within the observed energy range. ${ }^{3}$

In general we do not know which of the two $v_{m} G\left(v_{m}\right)$ terms in (28) dominates. Dropping the smaller of the two and using the bound (21) we arrive at

$$
\left|\int_{v_{m 1}}^{v_{m 2}} d v_{m} A_{\eta}^{\prime}\left(v_{m}\right)\right| \leq v_{e}\left|\sin \alpha_{\text {halo }}\right| \max \left[\bar{\eta}\left(v_{m 1}\right)-v_{m 1} \int_{v_{m 1}} d v \frac{\bar{\eta}(v)}{v^{2}}, v_{m 1} \rightarrow v_{m 2}\right] .
$$

Assumption 2 allows for arbitrary directions of the DM wind, therefore we need to use $\left|\sin \alpha_{\text {halo }}\right| \rightarrow 1$ above, while for Assumption 2 a one has $\sin \alpha_{\text {halo }} \simeq 0.5$. Note that a $\operatorname{sign}$ flip of $A_{\eta}^{\prime}$ will lead to cancellations in the integral on the l.h.s. of eq. (29) and make the bounds weaker. An observation of such a sign flip in the modulation amplitude would be a strong experimental evidence that the modulation is due to a DM signal. If such a sign flip is observed, one might be able to obtain stronger constraints by applying the bound for the region below and above the sign flip separately, to avoid the cancellations.

At present there is no indication of such a sign flip in which case the bound (29) simplifies to

$$
\int_{v_{m 1}}^{v_{m 2}} d v_{m} A_{\eta}^{\prime}\left(v_{m}\right) \leq v_{e}\left[\bar{\eta}\left(v_{m 1}\right)-v_{m 1} \int_{v_{m 1}} d v \frac{\bar{\eta}(v)}{v^{2}}\right] \quad \text { (Assumption 2) }
$$

and

$$
\int_{v_{m 1}}^{v_{m 2}} d v_{m} A_{\eta}^{\prime}\left(v_{m}\right) \leq 0.5 v_{e}\left[\bar{\eta}\left(v_{m 1}\right)-v_{m 1} \int_{v_{m 1}} d v \frac{\bar{\eta}(v)}{v^{2}}\right] \quad \text { (Assumption 2a). }
$$

The bounds (22), (30), (31) are the central results of this paper. In the following we will refer to them as

$$
\begin{array}{lll}
\text { Eq. (22) } & \text { "general bound" } & \text { (Assumption 1), } \\
\text { Eq. (30) } & \text { "symmetric halo" } & \text { (Assumption 2), } \\
\text { Eq. (31) } & \text { "symmetric halo, sin } \alpha=0.5 " & \text { (Assumption 2a). }
\end{array}
$$

The term "symmetric halo" should be understood in the sense of eqs. (25) and (26).

For completeness let us also mention an unintegrated bound, even though we will not use it for the numerical analysis. Using eq. (24) we have for the modulation amplitude

$$
A_{\eta}\left(v_{m}\right)=v_{e}\left|\sin \alpha_{\text {halo }}\right|\left|v_{m} g\left(v_{m}\right)-G\left(v_{m}\right)\right| .
$$

Note that the minus sign between the two terms is conserved, while in the general case, eq. (19), one is forced to sum the two terms in the bound. From eqs. (20) and (21) one then obtains the following bound on the modulation

$$
A_{\eta}\left(v_{m}\right) \leq v_{e}\left|\sin \alpha_{\text {halo }}\right| \max \left[\left|\frac{d \bar{\eta}}{d v_{m}}\right|, \frac{\bar{\eta}\left(v_{m}\right)}{v_{m}}-\int_{v_{m}} d v \frac{\bar{\eta}(v)}{v^{2}}\right] .
$$

\footnotetext{
${ }^{3}$ Note that the lowest energy bin in DAMA shows a somewhat smaller modulation amplitude, which might be an indication of a phase shift below the threshold.
} 
A similar bound has been obtained recently in [18]. If eq. (B4) of [18] is expanded in $u$ our bound (33) is obtained, if the derivative term dominates. However, eq. (B4) of [18] seems to neglect the possibility that the second term in eq. (33) dominates. This assumption is justified if data show no phase flip in the modulation and correspond to $v_{m}$ above the last phase flip $\left(A_{\eta}^{\prime} \geq 0\right)$. In this case eq. (33) becomes

$$
A_{\eta}^{\prime}\left(v_{m}\right) \leq-v_{e} \sin \alpha_{\text {halo }} \frac{d \bar{\eta}}{d v_{m}} .
$$

Note that this bound is complementary to the ones from eqs. (30), (31). By taking the integral over the modulation amplitude those bounds probe global properties of the amplitude over the considered energy range, whereas eq. (34) bounds the local size of the modulation amplitude at a given value of $v_{m}$. Both bounds are necessary conditions which a modulation signal with a DM origin has to fulfill.

\section{APPLYING THE BOUND TO DATA}

In this section we show how the bounds $(22),(30),(31)$ obtained in $v_{m}$ space for halo integrals can be applied to observable quantities, the unmodulated rate $\bar{R}$ and the amplitude of the modulation of the rate, $A_{R}$. The halo integral and the DM scattering rate are proportional to each other, see eq. (6). The relation is complicated by the fact that experiments typically do not observe the recoil energy $E_{n r}$ directly. The nuclear recoil energy is related to the observed energy through a quenching factor $Q$. In the case of CoGeNT and DAMA the observed energy $E_{e e}$ is measured in electron equivalent and is related to the recoil energy by $Q=E_{e e} / E_{n r}$. In general this is a nonlinear equation, as $Q$ also depends on the recoil energy. Finally, in an experiment data is reported in bins, and the continuous bounds derived above have to be integrated over the bin sizes. We relegate the details of the derivation to appendix $\mathrm{B}$, and quote below only the final results.

\subsection{Single target detector}

Let us first assume that the target consists of a single material, as is for instance the case for CoGeNT. We denote the modulation amplitude and the unmodulated rate in bin $i$ as $A_{i}$ and $R_{i}$, respectively, both in units of counts/day $/ \mathrm{kg} / \mathrm{keV}_{e e}$. The general bound (22) then becomes

$$
A_{i} \leq v_{e}\left[R_{i}\left(\alpha_{i}+\beta_{i}\right)-R_{i+1} \alpha_{i+1}^{\prime}-\langle\kappa\rangle_{i} \sum_{j=i}^{N} R_{j} \gamma_{j}\right] \quad \text { (Assumption 1) }
$$

and the bounds for the symmetric halo are

$$
\sum_{j=i}^{N} A_{j} x_{j} \leq v_{e} \sin \alpha\left[R_{i} y_{i}-\left\langle v_{m}\right\rangle_{i} \sum_{j=i+1}^{N} R_{j} \gamma_{j}\right] \quad \text { (Assumptions 2, 2a), }
$$

where the bounds (30) (Assumption 2) and (31) (Assumption 2a) are obtained for $\sin \alpha=1$ and 0.5 , respectively. The bin index $i$ runs from 1 to $N$, while the rates $R_{i}$ are zero for 
$i>N$. The coefficients $\alpha_{i}, \alpha_{i}^{\prime}, \beta_{i},\langle\kappa\rangle_{i}, \gamma_{i}, x_{i}, y_{i},\left\langle v_{m}\right\rangle_{i}$ are given in eqs. (B9) and (B12). They are known quantities calculable in terms of the form factor $F\left(E_{n r}\right)$, quenching factor $Q\left(E_{n r}\right)$, and Jacobians needed for changing the variable from $v_{m}$ to $E_{e e}$. They depend on the DM mass $m_{\chi}$ via the reduced mass $\mu_{\chi A}$, eq. (2), needed to convert $v_{m}$ into $E_{n r}$. The dependence on the scattering cross section and the local DM density is encoded in the constant factor $C$, eq. (6). This factor cancels completely, as expected, since it is a common factor for the modulation as well as for the rate.

The rates $R_{i}$ in the bounds (35) and (36) are the unmodulated scattering rate induced by DM without including any backgrounds. In a "background free" experiment, where the full observed event rate is due to DM, the bounds can be applied as they are. Here we want to be more conservative, and consider also the situation where an unknown background may contribute to the unmodulated rate. The remaining assumption is then just that the background itself is not modulated, only the DM signal. In each bin a fraction $\omega_{i}$ of the observed count rate $\mathcal{R}_{i}$ is due to DM, i.e.,

$$
R_{i}=\mathcal{R}_{i} \omega_{i}, \quad 0 \leq \omega_{i} \leq 1,
$$

so that we can replace $R_{i} \rightarrow \mathcal{R}_{i} \omega_{i}$ in eqs. (35) and (36) to obtain,

$$
\begin{aligned}
& A_{i} \leq v_{e}\left[\mathcal{R}_{i} \omega_{i}\left(\alpha_{i}+\beta_{i}\right)-\mathcal{R}_{i+1} \omega_{i+1} \alpha_{i+1}^{\prime}-\langle\kappa\rangle_{i} \sum_{j=i}^{N} \mathcal{R}_{j} \omega_{j} \gamma_{j}\right] \quad \text { (Assumption 1) }, \\
& \sum_{j=i}^{N} A_{j} x_{j} \leq v_{e} \sin \alpha\left[\mathcal{R}_{i} \omega_{i} y_{i}-\left\langle v_{m}\right\rangle_{i} \sum_{j=i+1}^{N} \mathcal{R}_{j} \omega_{j} \gamma_{j}\right] \quad \text { (Assumptions 2, 2a). }
\end{aligned}
$$

Now we have to find a set of $\omega_{i}(i=1, \ldots, N)$, such that the bound becomes the "weakest". In the following we describe two different procedures for this task. Procedure 1 is easy to implement but gives slightly weaker bounds, whereas procedure 2 involves an optimization algorithm but gives somewhat more stringent bounds.

Procedure 1: We can sum eq. (38) from bin $i$ to $N$ and drop the last term in the square bracket, since $\langle\kappa\rangle_{i}$ and $\gamma_{i}$ are positive:

$$
\sum_{j=i}^{N} A_{j} \leq v_{e}\left[\mathcal{R}_{i} \omega_{i} \alpha_{i}+\sum_{j=i}^{N} \mathcal{R}_{j} \omega_{j} \beta_{j}\right] \leq v_{e}\left[\mathcal{R}_{i} \alpha_{i}+\sum_{j=i}^{N} \mathcal{R}_{j} \beta_{j}\right] \quad \text { (Assumption 1) }
$$

where the last inequality was obtained by setting $\omega_{i}=1$. Similarly we can drop the second term from eq. (39) and obtain

$$
\sum_{j=i}^{N} A_{j} x_{j} \leq v_{e} \sin \alpha \mathcal{R}_{i} \omega_{i} y_{i} \leq v_{e} \sin \alpha \mathcal{R}_{i} y_{i} \quad \text { (Assumptions 2, 2a), }
$$

where the last inequality holds for $y_{i}>0 .{ }^{4}$ These bounds have to be satisfied for all bins $i$.

\footnotetext{
${ }^{4}$ According to eq. (B12) $y_{i}$ can also become negative. In that case the bound (41) is always violated for any $\omega_{i} \geq 0$.
} 
Procedure 2: A somewhat stronger bound can be obtained by searching for the optimal choice for the $\omega_{i}$, without dropping the last terms in eqs. (38) and (39). We present here a method based on a least-square minimization (an alternative procedure is outlined in appendix C). Let us denote the r.h.s. of eqs. (38) and (39) as $B_{i}$ which are functions of $\omega_{j}$. Then we can construct from eq. (38) the following least-square function

$$
X^{2}=\sum_{i=1}^{N}\left(\frac{A_{i}-B_{i}}{\sigma_{i}^{A}}\right)^{2} \Theta\left(A_{i}-B_{i}\right) \quad \text { (Assumption 1), }
$$

where $\sigma_{i}^{A}$ is the $1 \sigma$ error on $A_{i}$ (errors on $B_{i}$ are typically much smaller and we neglect them here). This $X^{2}$ can now be minimized with respect to $\omega_{j}$ under the condition $0 \leq \omega_{j} \leq 1$. The $\Theta$ function takes into account that there is only a contribution to $X^{2}$ if the bound is violated. Hence, $X^{2}$ will be zero if the bound is satisfied for all bins. A non-zero value of $X^{2}$ indicates that the bound is violated for some bin(s), weighted by the corresponding error in the usual way. In the case of eq. (39) one has to take into account that the modulation amplitude in each bin is used several times, leading to correlated errors for the l.h.s.:

$$
X^{2}=\sum_{i, j=1}^{N}\left(\mathcal{A}_{i}-B_{i}\right) S_{i j}^{-1}\left(\mathcal{A}_{j}-B_{j}\right) \Theta\left(\mathcal{A}_{i}-B_{i}\right) \Theta\left(\mathcal{A}_{j}-B_{j}\right) \quad \text { (Assumption 2,2a), }
$$

where

$$
\mathcal{A}_{i} \equiv \sum_{k=i}^{N} A_{k} x_{k} \quad \text { and } \quad S_{i j}=\sum_{k=1}^{N} \frac{d \mathcal{A}_{i}}{d A_{k}} \frac{d \mathcal{A}_{j}}{d A_{k}}\left(\sigma_{k}^{A}\right)^{2}=\sum_{k=\max (i, j)}^{N}\left(x_{k} \sigma_{k}^{A}\right)^{2} .
$$

While non-zero values of the $X^{2}$ functions (42) and (43) can be considered as a qualitative measure for the violation of the bound, the precise distribution of them should be determined by Monte Carlo studies. From the definition one can expect, however, that they will be approximately $\chi^{2}$ distributed if the bound is violated.

\subsubsection{CoGeNT}

Let us consider now the modulation signal reported by the CoGeNT experiment at $2.8 \sigma$ [4]. It has been pointed out that for specific assumptions on the halo (standard Maxwellian) there is a tension between the modulated and unmodulated rate in CoGeNT [20, 22, 23]. Recent analyses on the CoGeNT modulation signal can be found in refs. [24, 25], see also [18]. Here we use the CoGeNT data for a case study and apply the above bounds assuming spin-independent elastic scattering. For the germanium quenching factor we use $E_{e e}[\mathrm{keV}]=0.199\left(E_{n r}[\mathrm{keV}]\right)^{1.12}[26]$. We adopt the Helm parameterization of the spin-independent form factor, $F\left(E_{n r}\right)=3 e^{-q^{2} s^{2} / 2}[\sin (q r)-q r \cos (q r)] /(q r)^{3}$, with $q^{2}=2 m_{A} E_{n r}$ and $s=1 \mathrm{fm}, r=\sqrt{R^{2}-5 s^{2}}, R=1.2 A^{1 / 3} \mathrm{fm}$. We use the data from fig. 6 of ref. [20] where the total rate $\mathcal{R}$ and the modulation amplitude $A_{R}$ are given in four bins of $E_{e e}$ between 0.5 and $3.1 \mathrm{keV}$. We reproduce the data in table I. ${ }^{5}$

\footnotetext{
${ }^{5}$ We thank Mariangela Lisanti for providing us the data from fig. 6 of ref. [20].
} 


\begin{tabular}{c|c|c|c|c}
\hline \hline$E_{e e}$ bins [keV] & Mod. (Ass. 1, 2) & Mod. (Ass. 2a) & Unmod. rate & Corrected rate \\
\hline $0.5-0.9$ & $1.41 \pm 0.79$ & $0.90 \pm 0.72$ & $12.33 \pm 0.52$ & $5.29 \pm 0.52$ \\
$0.9-1.5$ & $0.84 \pm 0.59$ & $0.37 \pm 0.55$ & $4.33 \pm 0.39$ & $3.36 \pm 0.39$ \\
$1.5-2.3$ & $0.46 \pm 0.24$ & $0.48 \pm 0.22$ & $2.76 \pm 0.16$ & $2.76 \pm 0.16$ \\
$2.3-3.1$ & $0.66 \pm 0.24$ & $0.27 \pm 0.23$ & $2.83 \pm 0.17$ & $2.83 \pm 0.17$ \\
\hline
\end{tabular}

TABLE I: CoGeNT data on modulation amplitude and unmodulated rate [4] in $\mathrm{cnts} / \mathrm{day} / \mathrm{kg} / \mathrm{keV}$, as reported in . 6 of ref. [20]. The modulation has been extracted allowing for indpendent phases in each bin (Ass. 1) and for a constant but arbitrary phase (Ass. 2) (which lead to very similar amplitudes), and by requiring the maximum at June 2nd (Ass. 2a). In the last column we show the preliminary surface events corrected unmodulated rate [21].

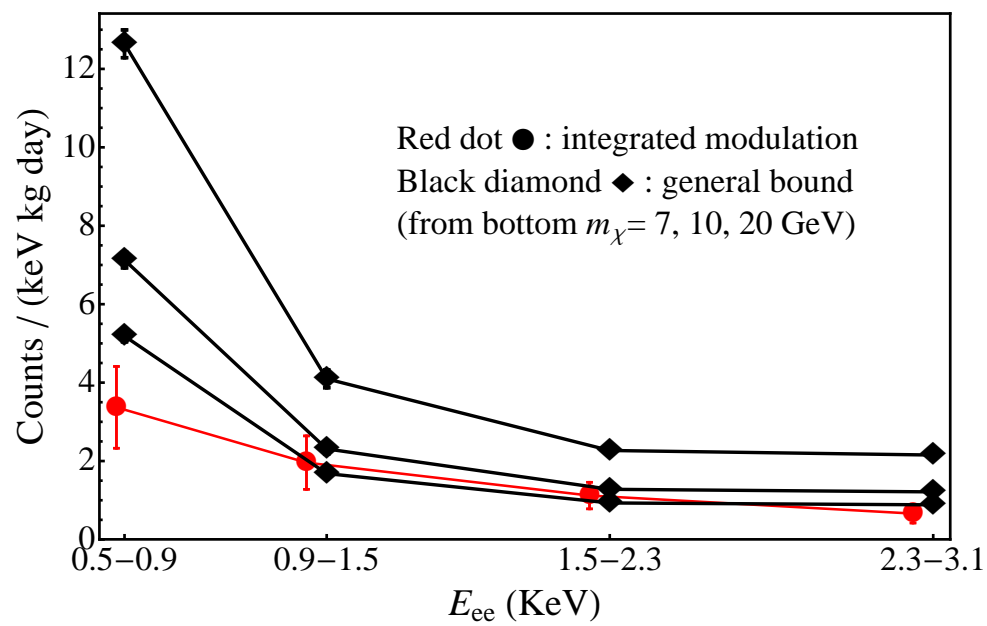

FIG. 1: Procedure 1 upper bound compared to the integrated modulation amplitude from the CoGeNT data, from fig. 6 of [20]. The red dots correspond to the l.h.s. of eq. (40). The black diamonds correspond to the upper bound obtained under Assumption 1, r.h.s of eq. (40), for DM masses of $m_{\chi}=7,10,20 \mathrm{GeV}$ (from bottom to top). The bins on the horizontal axis indicate the bin $i$ from which we start to sum the data. Error bars correspond to $1 \sigma$.

The modulation amplitude has been extracted in three different ways in [20]. First, by fitting independently the modulation phase for each energy bin, second, by assuming a constant phase for all bins, and third, by fixing the phase such that the modulation maximum is on June 2nd. These are precisely the requirements corresponding to our Assumptions 1, 2 , 2a, respectively. We can thus use the appropriate data on the modulation for each of the three assumptions. The modulation amplitudes in the first and the second case are very similar, while in the third case (forcing the phase to equal June 2nd) the amplitudes are lower and error bars are larger.

Choosing three values of the DM mass as examples, we show in fig. 1 the bound on the integrated modulation amplitude for Assumption 1, and using procedure 1. The bin labels on the horizontal axis give the $i$ th energy bin, which is the lower limit of summation in eq. (40). The data on amplitudes are shown as red dots, while the bounds are shown in black. Whenever a red dot lies above one of the bounds (within errors), the DM hypothesis 


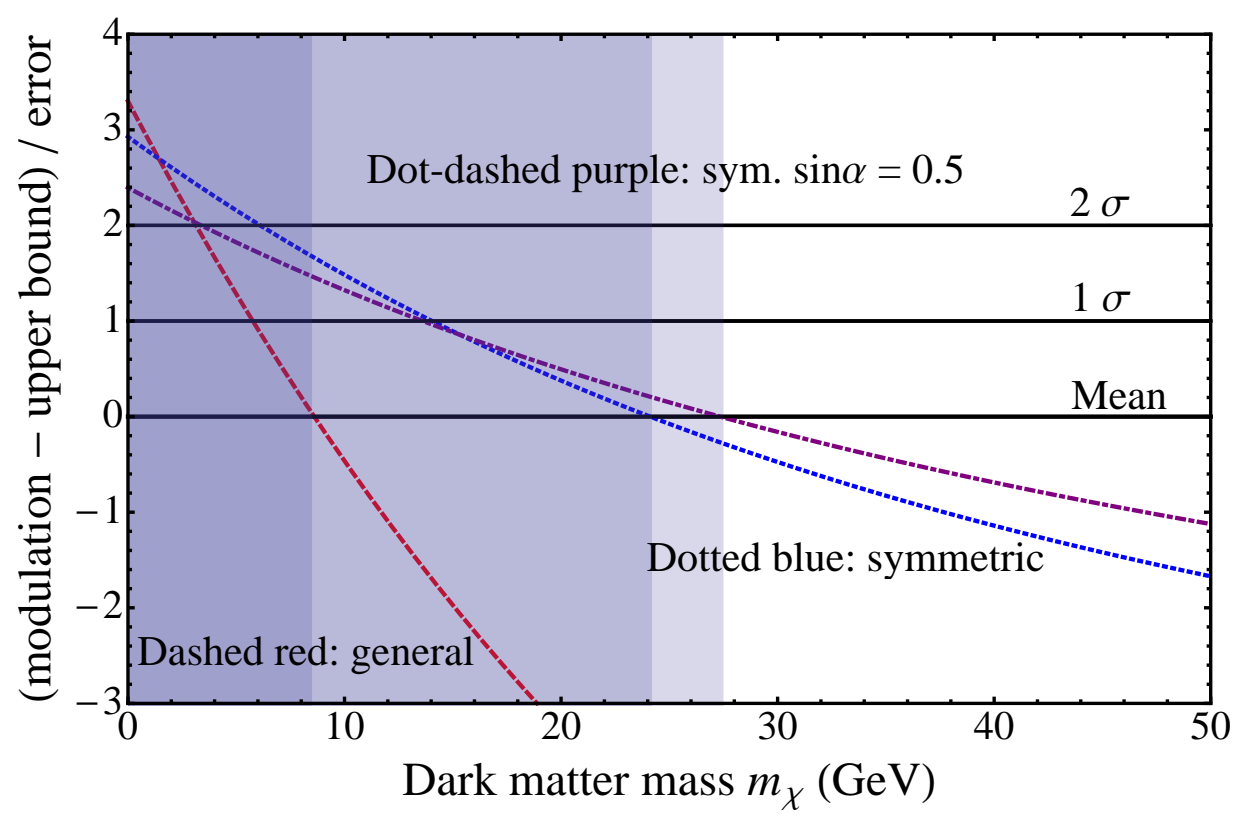

FIG. 2: Bounds on the CoGeNT modulation amplitude for the three assumptions about the DM halo (1, 2, 2a), and using eqs. (40) and (41) (procedure 1). We show the l.h.s. (integrated modulation amplitude) minus the r.h.s. (upper bound) divided by the error on the l.h.s., as a function of DM mass. We sum the data starting from bin $i=3,2$ and 3 for Assumptions 1, 2 and 2a, respectively. The bounds are violated in the regions where the curves are above zero, which are shaded in the plot.

is disfavoured. In fig. 1 this happens for the case of DM mass $m_{\chi}=7 \mathrm{GeV}$. Such light DM thus cannot be the source of the modulation, even under the very general assumption on the DM halo adopted here.

We see from fig. 1 that the strongest restriction comes from the bins $i=2$ or 3 . In fig. 2 we show the constraints on DM mass that follow from the bounds on the modulation amplitudes in these two bins. The bounds are obtained from procedure 1 for the three Assumptions 1, 2, 2a. We observe that the bound becomes stronger for smaller DM masses. This behaviour can be understood from how the coefficients defined in appendix B depend on the reduced mass $\mu_{\chi A}$. The bounds on amplitudes are violated for DM masses below $10,27,33 \mathrm{GeV}$, respectively, for the three assumptions. The lower bounds on $m_{\chi}$ are summarized in table II (left part), where we also give the bounds at $1 \sigma$ and $2 \sigma$. Notice that due to the smaller modulation amplitudes and larger errors when extracted from the data under Assumption 2a, at 95\% CL the corresponding bound becomes weaker than the one from Assumption 2 and equal to the one from Assumption 1, although naively one would expect the opposite. This can be traced back to the fact that, under Assumption 2a, the modulation phase is forced to take the value of June 2 nd which is not the one preferred by the data. The extracted modulation signal then gets weaker and consequently the bounds are more easily satisfied. We have also checked that bounds for Assumptions 2, 2a derived from eq. (34) give always weaker limits than the ones discussed here, which are based on eq. (30) and eq. (31).

In fig. 3 we show the $X^{2}$ functions according to procedure 2 as a function of the DM mass. For a given value of $m_{\chi}$ we minimize $X^{2}$ numerically with respect to the $\omega_{j}$. The conclusion 


\begin{tabular}{|c|c|c|c|c|c|c|c|c|}
\hline & \multicolumn{4}{|c|}{ unmodulated rate from $[4]$} & \multicolumn{4}{|c|}{ corrected unmod. rate $[21]$} \\
\hline & Mean & $68 \%$ & $95 \%$ & $X^{2} \leq 1$ & Mean & $68 \%$ & $95 \%$ & $X^{2} \leq 1$ \\
\hline Ass. 1: general bound & 8.5 & 6 & 3 & 7.3 & 10 & 6.5 & 3 & 10 \\
\hline Ass. 2: symmetric halo & 24 & 14 & 6 & 18 & 43 & 25 & 12.5 & 37 \\
\hline Ass. 2a: sym. halo, $\sin \alpha=0.5$ & 27.5 & 13.5 & 3.5 & 16 & 59.5 & 23 & 3 & 35 \\
\hline
\end{tabular}

TABLE II: Lower bounds on the DM mass in GeV, from the requirement that the modulation amplitude in CoGeNT is consistent with the upper bound from the unmodulated rate, according to the Assumptions 1, 2, 2a on the DM distribution. The bounds are obtained from procedure 1, requiring that eqs. (40) or (41) are satisfied for the mean value, or the $68 \%$ and $95 \%$ CL limits. The bounds labeled " $X^{2} \leq 1$ " are obtained from procedure 2 by requiring that $X^{2}$ defined in eqs. (42) or (43) is less than 1 . In the left part of the table we use the published unmodulated event rates from [4], whereas for the right part of the table we adopt the preliminary results on surface events contamination at low energies from [21].

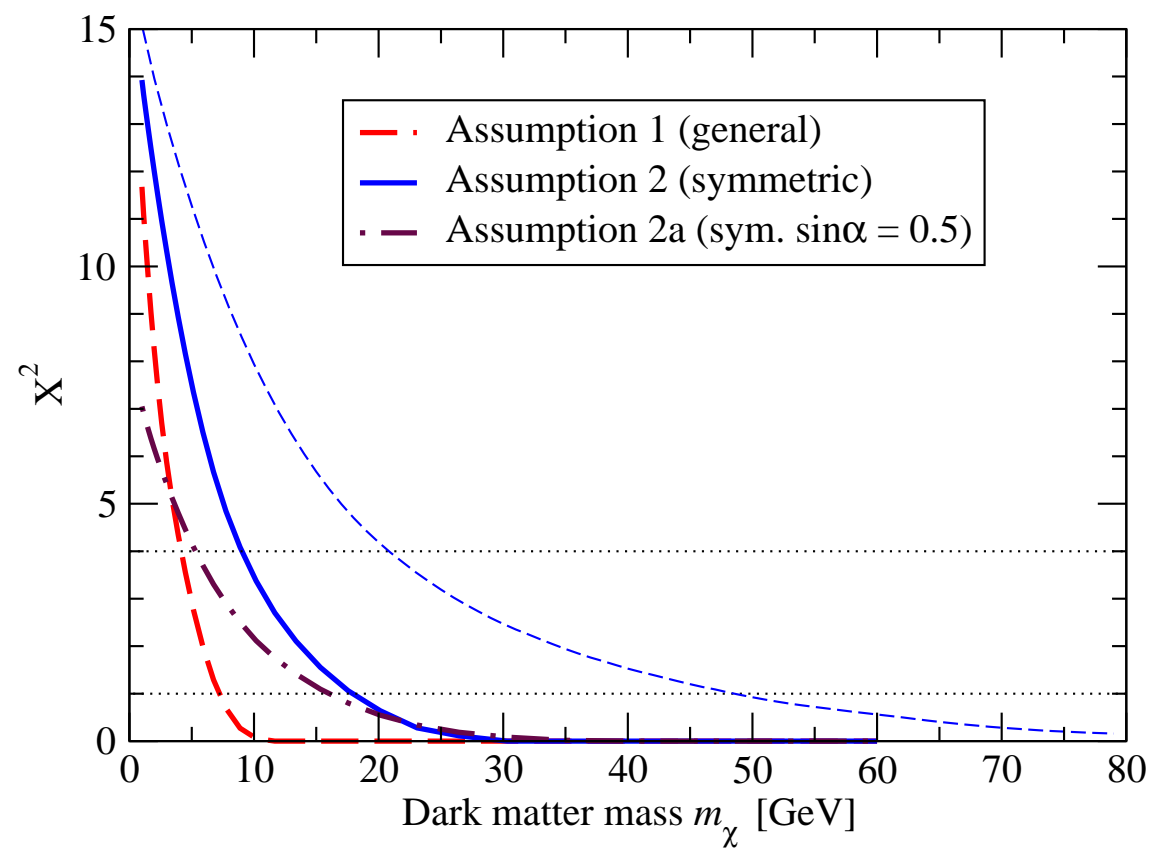

FIG. 3: Bounds on the CoGeNT modulation amplitude for the three assumptions about the DM halo (1, $2,2 \mathrm{a}$ ), always using procedure 2 . We show $X^{2}$ defined in eqs. (42) and (43) as a function of DM mass. The thin dashed curve is for illustrative purpose only; it corresponds to the data and errors on the modulation amplitude extracted with a free phase (Assumption 2) but using the bound for Assumption 2a, see text for details. If we assume that $X^{2}$ is distributed as a $\chi^{2}$ with 1 d.o.f. (see below) $X^{2}=1$ (4) corresponds to $68 \%$ (95\%) CL.

is similar to procedure 1, leading to similar lower bounds on the DM mass. Requiring that $X^{2} \leq 1$ one finds $m_{\chi} \geq 7.3,18,16 \mathrm{GeV}$ for Assumptions 1, 2, 2a, respectively. We observe again the unusual situation that Assumption 2a leads to a weaker constraint, due to the less significant signal for the modulation. To illustrate this effect we show in fig. 3 with a thin-dashed curve also $X^{2}$ that would follow from the hypothetical situation where the modulation amplitude would be as strong as in the case of Assumption 2. That is, we allow 

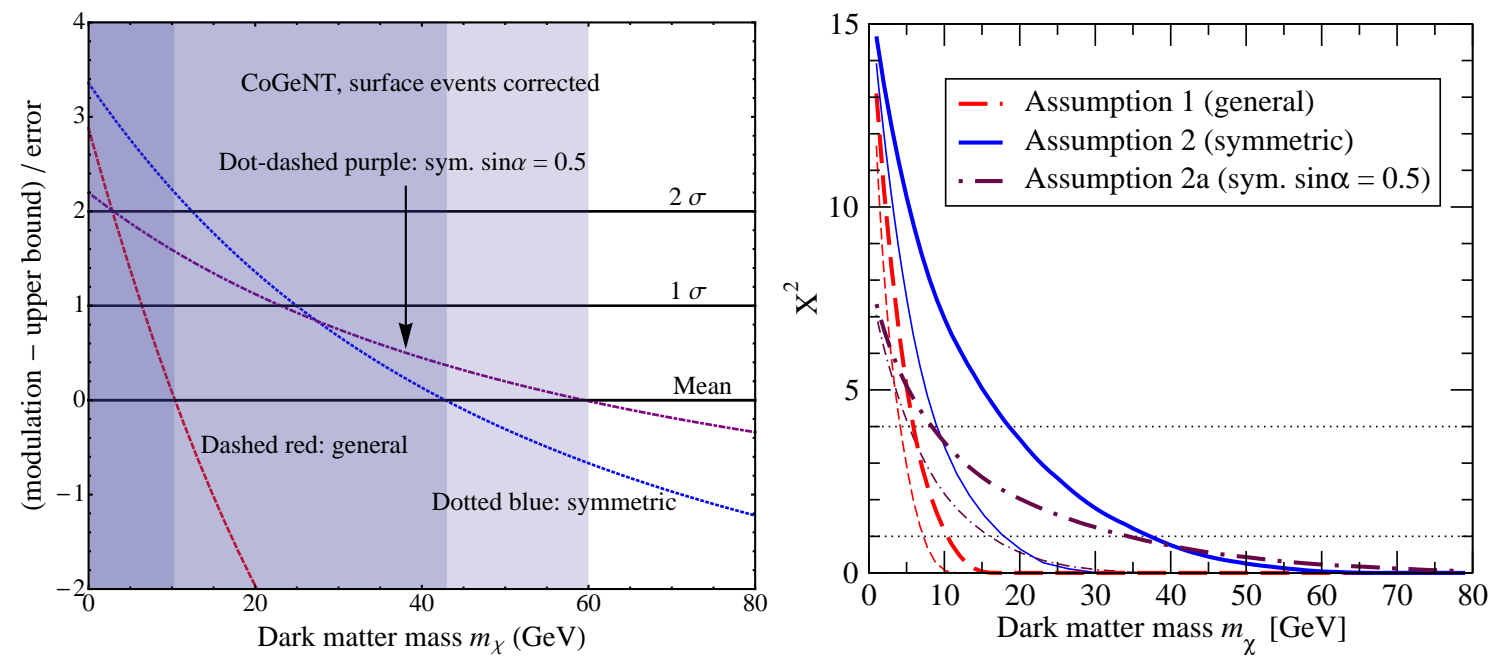

FIG. 4: Bounds on the CoGeNT modulation amplitude for the preliminary surface events corrected unmodulated rate [21] for the three assumptions about the DM halo (1, 2, 2a). The left plot shows the bounds according to procedure 1 , whereas the right plot shows $X^{2}$ defined in eqs. (42) and (43) (procedure 2). The thin curves in the right panel are the bounds without surface event subtruction reproduced from fig. 3 for the purpose of comparison with the thick curves obtain with the surface event corrected rates.

for an arbitrary energy independent phase, but assume that this phase turned out to be June 2nd (in contrast to the real CoGeNT data), so that we could apply the bound from Assumption $2 \mathrm{a}$ to these data. We see that in this case one would obtain a much stronger bound, disfavoring DM masses up to $60 \mathrm{GeV}$. This example shows the potential of our method in cases of a strong modulation signal in the data. If the signal for the modulation itself is weak, the bounds we derived will also give only weak constraints.

A recent re-analysis of CoGeNT data indicates that a significant fraction of the event excess at low energies could be due to surface events [21]. Assuming that surface events are not modulated, the unmodulated rate will be reduced after subtructing the surface events, while the modulation signal will remain, leading to a strengthening of our bounds. Here we estimate this effect by using the preliminary result for a surface events rejection efficiency shown on slide 19 of the presentation in [21] (red curve). Averaging this curve for the bins used in our analysis we find that the unmodulated rate in the first and second bins are reduced by a factor 0.43 and 0.78 , respectively, while the other two bins are not effected. This leads to the reduced event rates shown in the last column of tab. I. Fig. 4 shows the plots equivalent to figs. 2 and 3 but using the surface events corrected unmodulated rate. The general bound remains essentially the same. In this case the strongest limit (from procedure 1) for the uncorrected rate comes from summing data starting from bin 3 , whereas in the surface events corrected case it comes from bin 2, with only a minor reduction (79\%) of the rate, leading to a very similar limit. In contrast, for Assumptions 2 and 2a significantly stronger bounds are obtained, with the limit coming now from summing from bin 1 , with a $43 \%$ reduced rate. The surface events corrected bounds are summarized in the right part of table. II.

Due to the non-standard definition of the $X^{2}$ functions (42) and (43), involving the $\Theta$ function, the actual distribution of them is not clear a priori. Therefore we have performed 


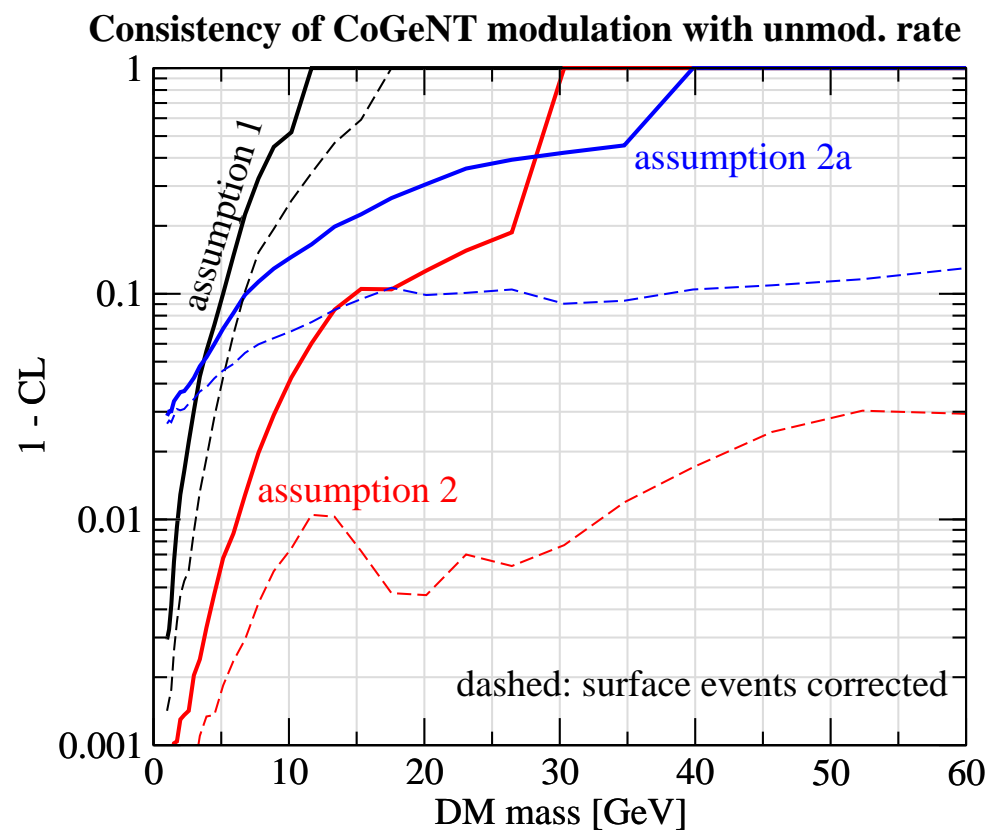

FIG. 5: The probability to obtain a $X^{2}$ larger than the one obtained from CoGeNT data, as determined by Monte Carlo simulation. Dashed curves correspond to surface event corrected data.

a Monte Carlo study in order to determine the distribution. For a given DM mass we first determine the optimal set of $\omega_{i}$ by minimizing the $X^{2}$. For those $\omega_{i}$ we assume that the bound is saturated and we simulate a large number of pseudo-data taking the r.h.s. of eqs. (38), (39) as mean value for a Gaussian with standard deviation given by the actual error on the modulation amplitude. ${ }^{6}$ For each random data set we calculate the $X^{2}$ value and obtain therefore the distribution of $X^{2}$ assuming that the bound is saturated. Then we can compare the $X^{2}$ of the actual data and calculate the probability of obtaining a $X^{2}$ larger than the observed one. Note that this procedure is conservative, since we assume that the mean value of the random data is the bound itself. If the "true" mean values are smaller than the bound, the distribution of $X^{2}$ would be shifted towards smaller values, leading to more stringent bounds.

We show the results of this calculation in fig. 5. The probabilities obtained in this way are in qualitative agreement with the numbers reported in tab. II. Under assumptions 2 and 2a, surface event corrected data is inconsistent with the DM hypothesis for any DM mass at the $97 \%$ and $90 \% \mathrm{CL}$, respectively. We find that if the bound is violated (i.e., for small DM masses) the $X^{2}$ distribution is close to a $\chi^{2}$-distribution with 1 d.o.f.. Large deviations from a $\chi^{2}$-distribution occur if $X^{2}$ is close to zero (i.e., for larger DM masses). In this case the $X^{2}$ distribution is strongly peaked at small values close to zero. This indicates that in such situations it is in most cases possible to find a set of $\omega_{i}$ such that $X^{2}$ becomes zero. This is the reason why non-trivial constraints are obtained under assumptions 2, 2a for

\footnotetext{
${ }^{6}$ In the case of Assumptions 2 and 2a we proceed iteratively. Starting from eq. (39) for $i=N$ we generate $A_{N}$, and then simulate successively $A_{i-1}$. This is necessary to obtain the correct random properties of the 1.h.s. of eq. (39).
} 
surface events corrected data at large masses, although the values of $X^{2}$ are relatively small, compare fig. 4.

\subsection{Bounds for multi-target experiments}

So far we have restricted the discussion to the situation where only one target nucleus is present, as for example Ge in CoGeNT. Let us now generalize our bounds to experiments where several elements are used as DM target. In this case both the modulation amplitude as well as the unmodulated rate will receive contributions from each element: $A_{i}=\sum_{n} A_{i}^{n}$ and $R_{i}=\sum_{n} R_{i}^{n}$, where $n$ labels the different target elements and $i$ energy bins. The bounds from procedure 1 hold for each of the elements separately, where in general all the coefficients appearing in the equations will depend on the nucleus type. Summing eqs. (40) and (41) over $n$ and using the fact that for positive $a_{i}$ and $b_{i}$, the following inequality holds, $\sum_{i} a_{i} b_{i} \leq\left(\sum_{i} a_{i}\right)\left(\sum_{j} b_{j}\right)$, we derive:

$$
\begin{aligned}
& \sum_{j=i}^{N} A_{j} \leq v_{e}\left[\mathcal{R}_{i} \sum_{n} \alpha_{i}^{n}+\sum_{j=i}^{N} \mathcal{R}_{j} \sum_{n}\left|\beta_{j}^{n}\right|\right] \quad \text { (Assumption 1) } \\
& \sum_{j=i}^{N} A_{j} \min _{n}\left(x_{j}^{n}\right) \leq v_{e} \sin \alpha \mathcal{R}_{i} \sum_{n}\left|y_{i}^{n}\right| \quad \text { (Assumptions 2, 2a). }
\end{aligned}
$$

On the l.h.s. of the last inequality it is necessary to take the minimum of $x_{j}^{n}$ between all the nuclei present in the target, for each bin. We used that $\alpha_{i}$ and $x_{j}$ are positive, see appendix B.

It is possible to generalize also the $X^{2}$ method to the case of multiple targets. For each nucleus the bounds (38) and (39) apply separately. We define the amplitude from element $n$ in the bin $i$ as $A_{i}^{n}=\epsilon_{i}^{n} A_{i}$, and similarly for the rate $R_{i}^{n}=\omega_{i}^{n} \mathcal{R}_{i}$. Then one can construct a $X^{2}$ similar to the one in the previous section, from l.h.s. minus r.h.s. of the following inequality:

$$
\epsilon_{i}^{n} A_{i} \leq v_{e}\left[\mathcal{R}_{i} \omega_{i}^{n}\left(\alpha_{i}^{n}+\beta_{i}^{n}\right)-\mathcal{R}_{i+1} \omega_{i+1}^{n} \alpha_{i+1}^{n}-\left\langle\kappa^{n}\right\rangle_{i} \sum_{j=i}^{N} \mathcal{R}_{j} \omega_{j}^{n} \gamma_{j}^{n}\right] \quad \text { (Assumption 1) }
$$

This $X^{2}$ is minimized over $\omega_{i}^{n} \geq 0, \epsilon_{i}^{n} \geq 0$ given the constraints $\sum_{n} \omega_{i}^{n} \leq 1$ and $\sum_{n} \epsilon_{i}^{n}=1$ for each $i$. A similar relation can be set up for Assumption 2.

\subsubsection{DAMA}

Let us now apply above bounds to the DAMA result, based on data from a NaI detector. DAMA observes a relatively large unmodulated count rate of about $1 \mathrm{cnt} / \mathrm{kg} / \mathrm{day} / \mathrm{keV}$ compared to the modulation amplitude of about $0.02 \mathrm{cnt} / \mathrm{kg} /$ day $/ \mathrm{keV}$. In contrast, CoGeNT reports a modulation amplitude in the range of 0.5 to $1 \mathrm{cnt} / \mathrm{kg} /$ day $/ \mathrm{keV}$, compared to an unmodulated rate of 3 to $4 \mathrm{cnt} / \mathrm{kg} / \mathrm{day} / \mathrm{keV}$. Because of this much smaller modulation amplitude compared to the unmodulated rate for DAMA we expect the signal to be consistent 

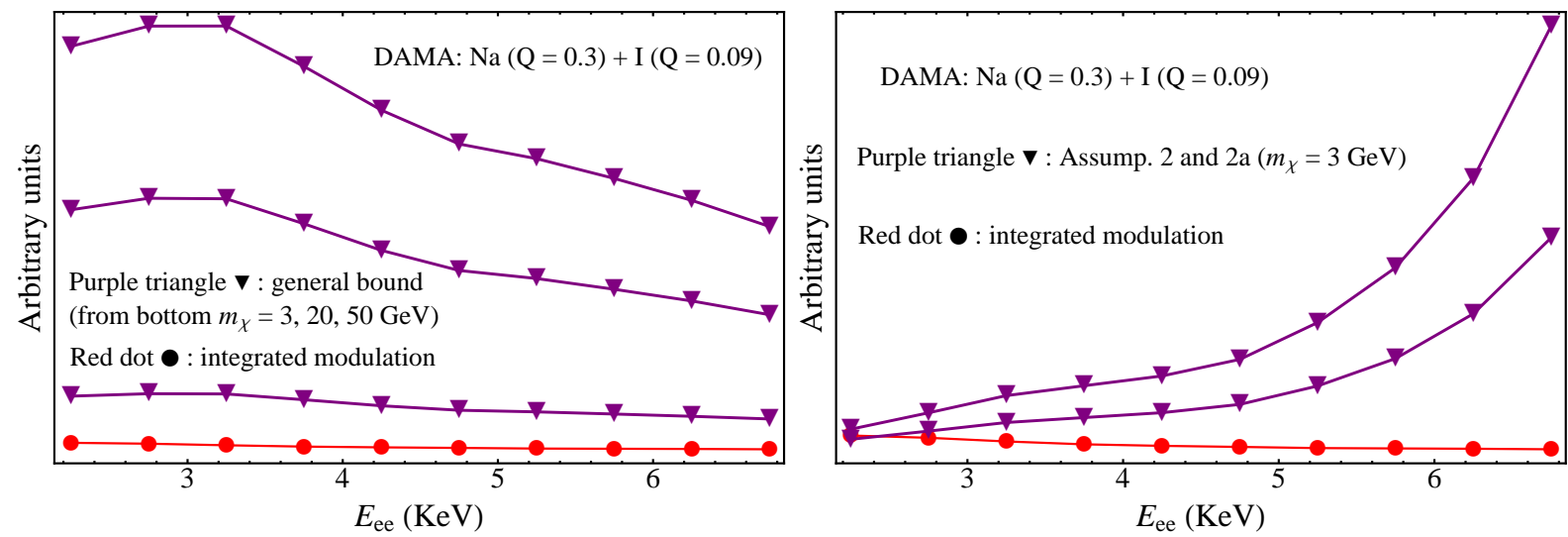

FIG. 6: Upper bound compared to the integrated modulation amplitude for DAMA data for Assumption 1 (left) and Assumptions 2 and 2a (right). We assume quenching factors $q=0.3$ and $q=0.09$ for $\mathrm{Na}$ and I, respectively. The red dots correspond to the l.h.s. of eqs. (45) and (46), and the purple triangles to the r.h.s.. In the left panel we show the bounds for DM masses of $m_{\chi}=3,20,50 \mathrm{GeV}$ (from bottom), in the right panel for $m_{\chi}=3 \mathrm{GeV}$. Bins are $0.5 \mathrm{keV}$ wide and we sum all bins starting from bin $i$ shown on the horizontal axis up to 7 keVee. Error bars are negligible and are not shown for clarity. All dark matter masses are compatible with the modulation.

with our bounds. It is also well known that a consistent fit of the DAMA modulated and unmodulated data is possible e.g., for a Maxwellian halo, although in certain parameter regions the unmodulated rate does provide a non-trivial constraint, see e.g. $[8,11]$. In fig. 6 we show the bounds for DAMA, assuming quenching factors of 0.3 for $\mathrm{Na}$ and 0.09 for I (constant in energy) using the spectral data on the modulated and unmodulated rates from [3]. We find that both the general bound, eq. (45), as well as the bounds for a symmetric halo, eq. (46), are consistent with the modulation even for a DM mass as small as $3 \mathrm{GeV}$. As noted above the bounds get weaker for larger DM masses, hence they do not place any relevant constraint on the dark matter interpretation of the DAMA modulation signal.

In deriving the multi-target bounds eqs. (45) and (46) we had to use inequalities related to the presence of several contributions to the rates, which makes the bounds somewhat weaker than single target bounds. In case of $\mathrm{NaI}$ we have a relatively large hierarchy between the two targets since $A=23$ for Na and $A=127$ for I. Therefore, one may consider also the situation that only one of them dominates the signal. If scattering on both targets is kinematically allowed, iodine will dominate because of the $A^{2}$ enhancement of the spinindependent scattering cross section (which we will assume here). For low DM masses, scattering on iodine may be kinematically forbidden since $v_{m}$ is larger than the escape velocity of the halo and in that case the signal is provided only by scattering off $\mathrm{Na}$ (this requires additional assumptions on the escape velocity, which in general are not necessary for our bounds). We show the bounds for Assumptions 2 and 2a assuming that scattering on either Na or I dominates in fig. 7 for some representative DM masses. In both cases only very weak constraints are obtained. While scattering on $\mathrm{Na}$ is consistent down to $m_{\chi} \approx 3 \mathrm{GeV}$ for Assumption 2a, scattering on iodine is consistent for $m_{\chi} \gtrsim 30 \mathrm{GeV}$ under Assumption 2. (Note that below around $30 \mathrm{GeV}$ typically scattering on $\mathrm{Na}$ dominates.)

As expected, we conclude that based solely on our bounds the DAMA signal is compatible 

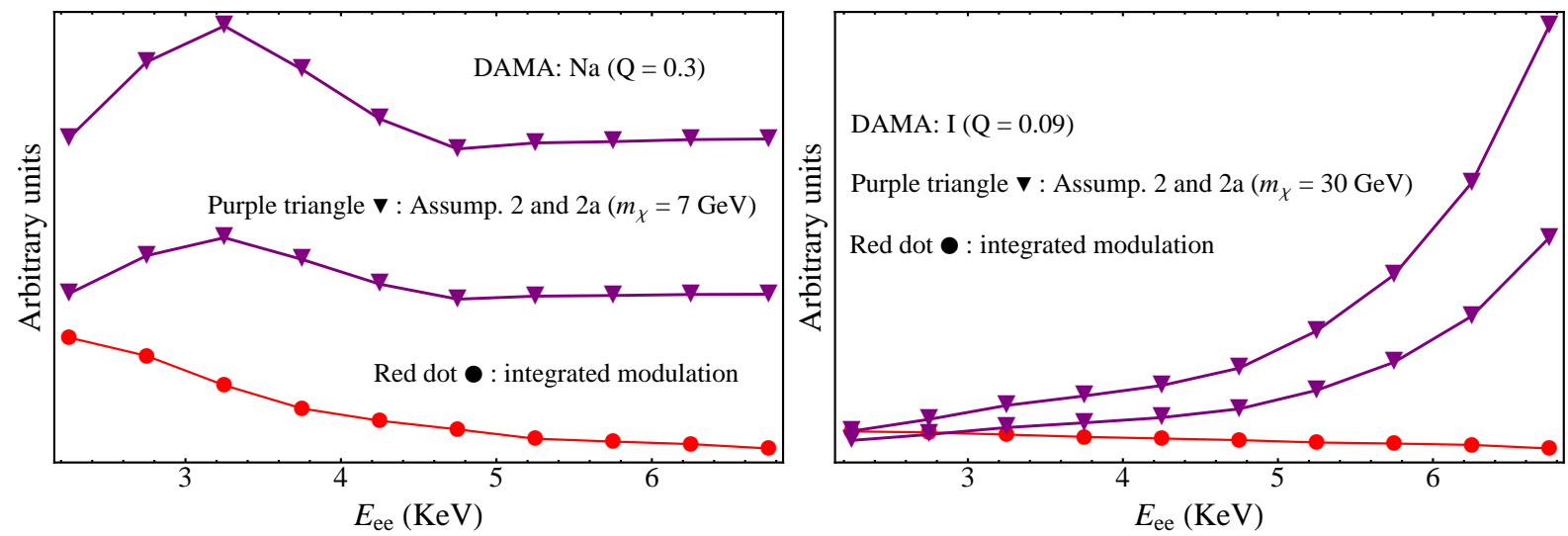

FIG. 7: Upper bound compared to the integrated modulation amplitude from DAMA data for Assumptions 2 and 2a, assuming that DM scatters only on sodium (left) or iodine (right). The red dots correspond to the 1.h.s. of eq. (41) and the purple triangles to the r.h.s., for a DM mass of $m_{\chi}=7 \mathrm{GeV}$ (left) and $30 \mathrm{GeV}$ (right). Bins are $0.5 \mathrm{keV}$ wide and we sum all bins starting from bin $i$ shown on the horizontal axis up to 7 keVee. Error bars are negligible and are not shown for clarity.

with assuming dark matter scattering being its origin. We do not expect that this conclusion will change significantly if bounds from procedure 2 according to eq. (47) were applied. Therefore, we limited ourselves to the discussion based on procedure 1 for DAMA.

\section{SUMMARY AND DISCUSSION}

The annual modulation is probably the most distinctive signature of dark matter and is playing (and will certainly play) a central role in revealing its existence and nature. However, it is not always clear that the modulation detected by a DM direct detection experiment is caused by the dark matter particles and not by any other unknown background source. In this work we have presented a consistency check for the amplitude of the modulation and the unmodulated count rate, which is a necessary (but not sufficient) condition for DM being the origin of an observed annual modulation.

We have derived upper bounds on the energy integrated modulation amplitude in terms of the unmodulated rate by expanding the DM velocity integral to first order in the earth's velocity $v_{e} \approx 30 \mathrm{~km} / \mathrm{s}$. It holds for a wide class of particle physics models where the differential scattering cross section $d \sigma / d E_{n r}$ is proportional to $1 / v^{2}$. Although we have only focused on elastic scattering the method can also be generalized to the inelastic case. The important aspect of our work is that our bounds hold for very general assumptions about the DM velocity distribution $f(\mathbf{v})$ in the sun's vicinity. We have presented bounds under the hypothesis of a single DM species and the following assumptions on the DM halo:

- Assumption 1, bound in eq. (22): We assume that the only time dependence is induced by the rotation of the earth around the sun. The halo itself is static on the time scale of months to years and spatially constant at the scale of the sun-earth distance. Otherwise the DM velocity distribution can have an arbitrary structure, including, for example, several streams coming from various directions. In order to 
saturate this bound the halo has to be very peculiar, with rather unrealistic properties.

- Assumption 2, bound in eq. (30): In addition to Assumption 1 we assume that there is just one preferred direction of the DM velocity distribution in the rest frame of the sun (independent of the minimal velocity $v_{m}$ in the halo integral). This requires certain symmetries of the velocity distribution, which are specified in eqs. (25) and (26). It covers typically situations where the DM distribution is dominated by one single component, which may come from an arbitrary (but constant) direction. Assumption 2 requires that the phase of the modulation is independent of the recoil energy (up to a phase flip of half a year).

- Assumption 2a, bound in eq. (31): We require that the preferred direction from Assumption 2 is aligned with the motion of the sun. This is fulfilled for any isotropic halo, and also for tri-axial halos up to corrections due to the peculiar velocity of the sun. Furthermore, it includes the possibility of streams parallel to the motion of the sun, such as a possible dark disk. Assumption 2 a requires that the maximum (or minimum) of the event rate is around June 2 nd.

The theoretical bounds are obtained in terms of the minimal velocity $v_{m}$ and have to be related to observable quantities by translating into recoil energy. We have outlined two possible procedures for this task in section 5 for the case of elastic scattering, taking into account statistical errors and the possibility that an unknown background may contribute to the unmodulated rate, but that it has no time-dependence. As an example we have applied the proposed consistency checks to the annual modulation signals reported by the CoGeNT and DAMA experiments. While DAMA data are compatible with a dark matter origin for its modulation, severe restrictions on the dark matter mass can be set for the case of CoGeNT. Applying our bounds we find that the CoGeNT modulation amplitude can be consistent with the unmodulated rate at the $68 \%$ CL only for DM masses $m_{\chi} \gtrsim 7.3,18,16 \mathrm{GeV}$ for the Assumptions 1, 2, 2a, respectively. If preliminary results on a possible surface events contamination of the unmodulated rate at low energies in CoGeNT are confirmed, those bounds would become even more restrictive. In this case, CoGeNT modulation data would be inconsistent with the DM hypothesis under assumptions 2 and 2a at about $97 \%$ and $90 \% \mathrm{CL}$, respectively. DAMA has a relatively large unmodulated count rate of about $1 \mathrm{cnt} / \mathrm{kg} /$ day $/ \mathrm{keV}$ compared to the modulation amplitude of about $0.02 \mathrm{cnt} / \mathrm{kg} / \mathrm{day} / \mathrm{keV}$. Therefore, our bounds are not very stringent and the modulation amplitude is consistent with the unmodulated rate. In contrast, CoGeNT reports a modulation amplitude in the range of 0.5 to $1 \mathrm{cnt} / \mathrm{kg} /$ day $/ \mathrm{keV}$, compared to an unmodulated rate of 3 to $4 \mathrm{cnt} / \mathrm{kg} / \mathrm{day} / \mathrm{keV}$. Because of this relatively large ratio between modulated and unmodulated rate our method provides stringent constraints in the case of CoGeNT. Several comments are in order:

( $i$ ) In deriving the bounds we assume a certain smoothness of the halo, since we are expanding in $v_{e}$. Spikes in the velocity distribution much narrower than $30 \mathrm{~km} / \mathrm{s}$ are not covered by our procedure.

(ii) Our bounds assume that a modulation signal is present in the data. If the significance of the modulation is weak, the bounds are more easily satisfied. This effect is explicitly illustrated in sec. 5 by using CoGeNT data under different assumptions regarding 
the modulation signal. The method discussed here cannot be used to establish the presence of a modulation, it can only test whether a given modulation signal is consistent with the unmodulated rate.

(iii) In this work we have used the relation between the minimal velocity $v_{m}$ and nuclear recoil energy $E_{n r}$ implied by elastic scattering, see eq. (2) and appendix B. The bounds can be generalised in a straightforward way also to inelastic scattering. However, in that case $v_{m}=\left(m_{A} E_{n r} / \mu_{\chi A}+\delta\right) / \sqrt{2 m_{A} E_{n r}}$ has a minimum in $E_{n r}$, and therefore there is no one-to-one correspondence between $v_{m}$ and $E_{n r}$. When translating from $v_{m}$ to $E_{n r}$ one has to take into account that different disconnected regions in $E_{n r}$ can contribute to a given interval in $v_{m}$.

(iv) The type of DM-nucleus interaction (i.e., the particle physics) has to be specified before applying our bounds. Apart from the $1 / v^{2}$ dependence of the differential cross section $d \sigma / d E_{n r}$, the $E_{n r}$ dependence of the interaction is encoded in the form factor $F\left(E_{n r}\right)$. It can describe conventional spin-independent or spin-dependent interactions, but also a possible non-trivial $q^{2}$-dependence of the DM-nucleus interaction, all of which can be absorbed into $F\left(E_{n r}\right)$.

To conclude, we have presented a consistency check for the amplitude of a DM induced annual modulation compared to the unmodulated event rate in a given DM direct detection experiment. Our bounds rely only on very mild and realistic assumptions about the DM halo. We believe that the proposed method is a useful check which any annual modulation signal should pass. A violation of our bounds suggests a non-DM origin of the annual modulation, or requires rather exotic properties of the DM distribution, for example very sharp spikes and edges. Such extreme features of the halo should have other observable consequences, such as surprising spectral features or strong energy dependence of the modulation phase. Such features could be used as additional diagnostics, beyond the signatures explored here, which are restricted to the energy integrated modulation amplitude, irrespective of the phase.

\section{Acknowledgments}

We thank Felix Kahlhöfer for useful comments on the manuscript. T.S. and J.Z. are grateful for support from CERN for attending the CERN-TH institute DMUH'11, 18-29 July 2011, where this work was initiated. The work of T.S. was partly supported by the Transregio Sonderforschungsbereich TR27 "Neutrinos and Beyond" der Deutschen Forschungsgemeinschaft. J.H.-G. is supported by the MICINN under the FPU program and would like to thank the division of M. Lindner at MPIK for very kind hospitality.

\section{Appendix A: Maxwellian halo}

As a cross check we apply our formalism to the commonly used Maxwellian velocity distribution, with a cut off at an escape velocity $v_{\text {esc }}$. In the galactic rest frame it is given by

$$
f_{\text {gal }}(\mathbf{v}) \propto\left[\exp \left(-\mathbf{v}^{2} / \bar{v}^{2}\right)-\exp \left(v_{\text {esc }}^{2} / \bar{v}^{2}\right)\right] \Theta\left(v_{\text {esc }}-v\right)
$$




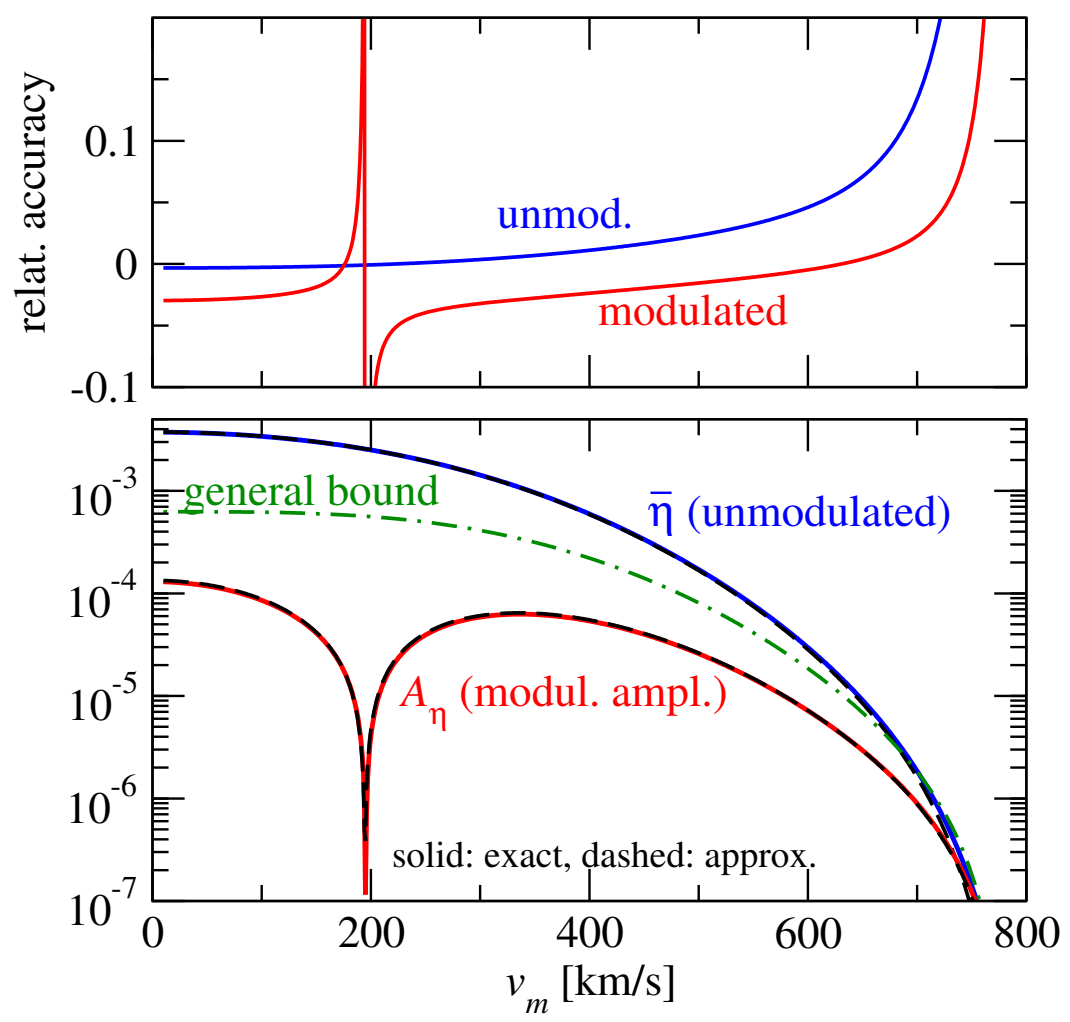

FIG. 8: Comparison for the Maxwellian halo of the exact (solid line) and $v_{e}$ expanded (dashed) modulated and unmodulated halo integrals $A_{\eta}$ and $\bar{\eta}$, respectively. The modulation flips the phase by half a year below the zero of the amplitude. In the lower panel the general bound (22) is also shown (dashed-dotted curve). The upper panel shows the relative accuracy defined as (exact - approx)/exact.

where we use $\bar{v}=220 \mathrm{~km} \mathrm{~s}^{-1}$ and $v_{\text {esc }}=550 \mathrm{~km} \mathrm{~s}^{-1}$. This distribution is boosted to the sun's and earth's rest frames as described in section 2 . For the above velocity distribution the halo integral $\eta\left(v_{m}, t\right)$ is known analytically. We define the "exact" modulated and unmodulated halo integrals as

$$
\begin{aligned}
\bar{\eta}^{\text {exact }}\left(v_{m}\right) & \equiv \frac{1}{2}\left[\eta\left(v_{m}, t_{*}\right)+\eta\left(v_{m}, t_{*}+0.5\right)\right], \\
A_{\eta}^{\text {exact }}\left(v_{m}\right) & \equiv \frac{1}{2}\left[\eta\left(v_{m}, t_{*}\right)-\eta\left(v_{m}, t_{*}+0.5\right)\right],
\end{aligned}
$$

where for the (exact) analytic expressions $\eta\left(v_{m}, t\right)$ we are are using the results given in the appendix of [27]. Above, $t_{*}$ is June 2 nd and $A_{\eta}^{\text {exact }}\left(v_{m}\right)=\left|A_{\eta}^{\prime \text { exact }}\left(v_{m}\right)\right|$.

We first check the accuracy of the $v_{e}$ expansion. Expanding to zeroth order in $v_{e}$ gives the approximate unmodulated rate (12), while the modulation amplitude is given by expanding to linear order in $v_{e},(32)$, with $\sin \alpha_{\text {halo }}=0.5$. The comparison with the "exact" expressions is shown in fig. 8. Both for the modulation amplitude and the unmodulated rate we find excellent agreement, with the differences hardly visible on logarithmic scale. As seen from the upper panel, for large regions of $v_{m}$ the agreement is within few \%, apart from the zero of the modulation amplitude. Minor deviations appear for large $v_{m}$ values, where non-linear effects due to the cut-off at the escape velocity become important. We see that our expression 

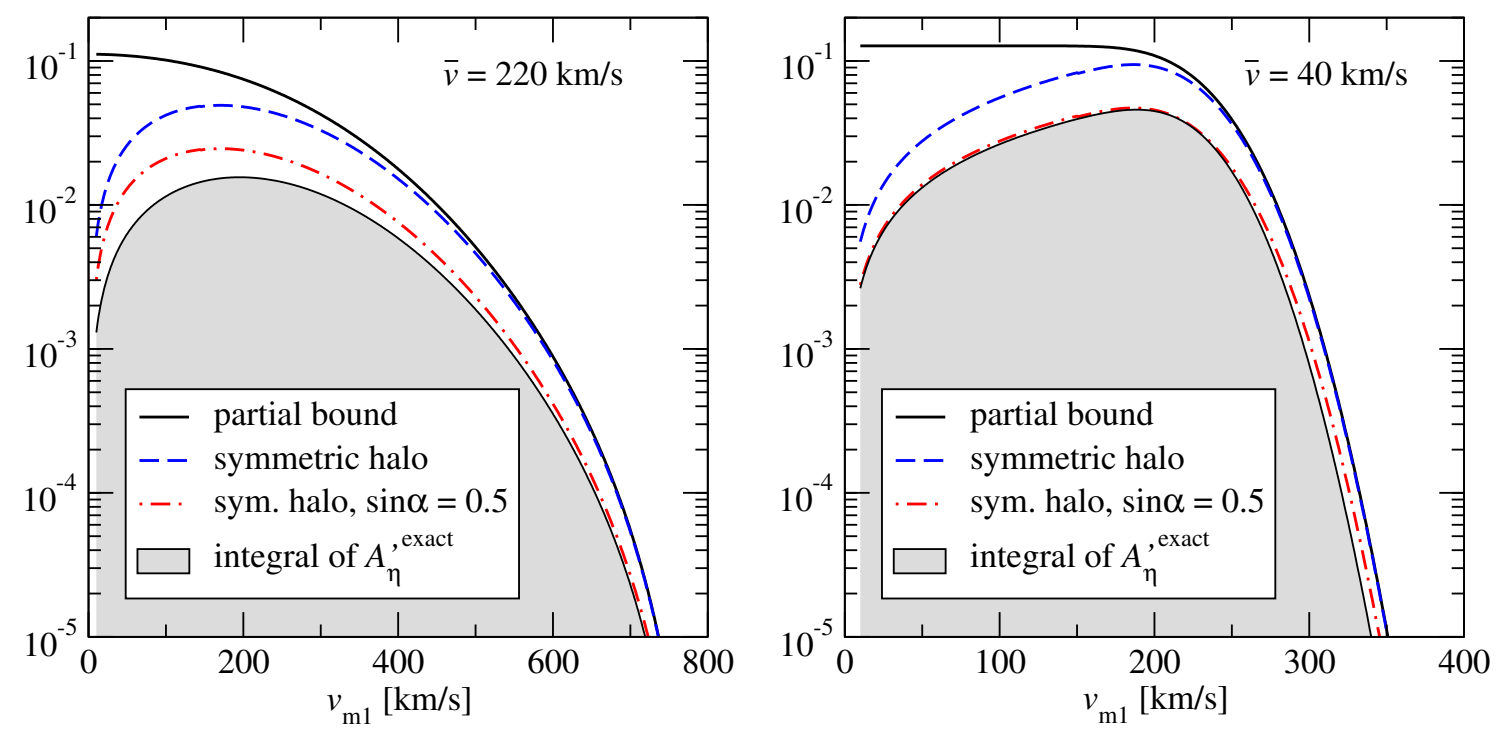

FIG. 9: The bounds on the modulation amplitude for a Maxwellian velocity distribution and velocity dispersions $\bar{v}=220 \mathrm{~km} / \mathrm{s}(40 \mathrm{~km} / \mathrm{s})$ in the left (right) panels. The shaded region shows the integral of $\int_{v_{m 1}}^{\infty} A_{\eta}^{\text {exact }}$. We show the bound for a "symmetric halo" from eq. (30), and the bound from eq. (31) which assumes that the DM wind is parallel to the motion of the sun ("sym. halo, $\sin \alpha=0.5$ "). The solid curve ("partial bound") shows the bound eq. (30), but without the second term in the square bracket.

for $A_{\eta}$ captures accurately the phase flip of the modulation. At low $v_{m}$ the $G\left(v_{m}\right)$ term in eq. (32) dominates, leading to the maximum of the count rate in December, the modulation amplitude becomes zero when the two terms cancel, and the maximum in June at large $v_{m}$ is obtained from the $g\left(v_{m}\right)$ term.

We compare next the bounds with the actual modulation amplitude. The dash-dotted (green) curve in fig. 8 shows the general bound, eq. (22), which follows from Assumption 1. Clearly, the Maxwellian halo is far from saturating this bound. Much tighter constraints are obtained from Assumptions 2 and 2a. Fig. 9 shows the bounds (30) and (31) on integrals of modulation amplitudes. The shaded region shows the l.h.s. of the bounds, $\int_{v_{m 1}} d v A_{\eta}^{\prime}$, where the upper boundary of the integration is chosen so high that the DM signal vanishes. Note that $A_{\eta}^{\prime}$ flips the sign at $v_{m} \sim 200 \mathrm{~km} / \mathrm{s}$, which explains the maximum of the integral around $200 \mathrm{~km} / \mathrm{s}$. The curve labeled "partial bound" corresponds to the bound following from Assumption 2, eq. (30), but without the second term in the square bracket. We find that it is only slightly more stringent than the general bound that follows from Assumption 1 , (22), especially for large $v_{m}$. Although it does not capture the behaviour at low $v_{m}$ it is rather similar to the full bound, eq. (30), (dashed curve) for $v_{m} \gtrsim 400 \mathrm{~km} / \mathrm{s}$. The bound from Assumption 2a, eq. (31) (dash-dotted curve), which assumes $\sin \alpha_{\text {halo }}=0.5$, is roughly a factor 2 larger than the prediction for small $v_{m 1}$ and approaches the true modulation around $v_{m 1} \gtrsim 400 \mathrm{~km} / \mathrm{s}$. In the right panel we show that for a Maxwellian distribution with very small dispersion $(\bar{v}=40 \mathrm{~km} / \mathrm{s})$ the bound for Assumption 2a is nearly saturated. As mentioned in sec. 3, the inequalities used to derive the bound (31) become equalities if the velocity distribution $f(\mathbf{v}) \propto \delta(\vartheta)$, which is approximated by the Maxwellian with small $\bar{v}$. 


\section{Appendix B: Translating bounds in $v_{m}$ space to observable quantities}

In sections 3 and 4 we have derived bounds involving the halo integral $\bar{\eta}\left(v_{m}\right)$ and the modulation amplitude $A_{\eta}\left(v_{m}\right)$. Here we give details on how to translate these bounds into bounds that involve observable rates as functions of nuclear recoil energy $E_{n r}$. The nuclear energy is measured in electron-equivalents, $E_{e e}$, which is related to $E_{n r}$ through a quenching factor $Q$. We also define a differential quenching factor $q$,

$$
q \equiv \frac{d E_{e e}}{d E_{n r}}=Q+E_{n r} \frac{d Q}{d E_{n r}}, \quad \text { where } \quad Q \equiv \frac{E_{e e}}{E_{n r}},
$$

which then gives the translation between $v_{m}$ and $E_{e e}$,

$$
v_{m}=\sqrt{\frac{m_{A} E_{e e}}{2 \mu_{\chi A}^{2} Q}}, \quad \frac{1}{\xi\left(E_{e e}\right)} \equiv \frac{d v_{m}}{d E_{e e}}=\frac{d v_{m}}{d E_{n r}} \frac{d E_{n r}}{d E_{e e}}=\frac{1}{2 \mu_{\chi A}} \sqrt{\frac{m_{A} Q}{2 E_{e e}}} \frac{1}{q} .
$$

The quenching factor $Q$ is in general energy dependent. If it is energy independent, then $q=Q$, while if data are reported directly in $E_{n r}$ then furthermore $q=Q=1$. Note that $q, Q, v_{m}, \xi$, are all positive.

The modulation amplitude and rate in units of counts/day $/ \mathrm{kg} / \mathrm{keV}$ ee are given as

$$
A_{R}\left(E_{e e}\right)=\kappa A_{\eta}\left(v_{m}\right), \quad \bar{R}\left(E_{e e}\right)=\kappa \bar{\eta}\left(v_{m}\right) \quad \text { with } \quad \kappa\left(E_{e e}\right) \equiv \frac{C F^{2}\left(E_{e e} / Q\right)}{q\left(E_{e e}\right)},
$$

with the constant $C$ defined in (6). To keep the notation simple we use the same symbols for rate and modulation whether they depend on recoil energy in $\mathrm{keV}_{e e}$ or $\mathrm{keV}_{n r}$. The units are denoted in the argument $\left(E_{e e}\right.$ versus $\left.E_{n r}\right)$ and should be clear from the context.

Let us consider first the general bound following from Assumption 1. Multiplying eq. (22) by $\kappa$ and converting the integral over $v_{m}$ into $E_{e e}$ by using eq. (B2) one finds

$$
A_{R}\left(E_{e e}\right) \leq v_{e}\left[-\frac{d(\bar{R} \xi)}{d E_{e e}}+\bar{R}\left(\frac{d \xi}{d E_{e e}}+\sqrt{\frac{2 \mu_{\chi A}^{2} Q}{m_{A} E_{e e}}}+\frac{\xi}{\kappa} \frac{d \kappa}{d E_{e e}}\right)-\kappa \int_{E_{e e}} d E_{e e} \frac{\bar{R}}{\xi \kappa} \frac{2 \mu_{\chi A}^{2} Q}{m_{A} E_{e e}}\right]
$$

Eq. (34) for Assumptions 2, 2a becomes similarly:

$$
A_{R}\left(E_{e e}\right) \leq v_{e} \sin \alpha_{\text {halo }}\left[-\frac{d(\bar{R} \xi)}{d E_{e e}}+\bar{R}\left(\frac{d \xi}{d E_{e e}}+\frac{\xi}{\kappa} \frac{d \kappa}{d E_{e e}}\right)\right]
$$

Note that the constant $C$ containing the cross section and local DM density drops out, as expected, since it is a common pre-factor for rate as well as modulation. In practise $A_{R}$ and $\bar{R}$ are given in bins in $E_{e e}$. Let us define the bin average of a quantity $X\left(E_{e e}\right)$ as

$$
\langle X\rangle_{i} \equiv \frac{1}{\Delta E_{i}} \int_{E_{i 1}}^{E_{i 2}} d E_{e e} X\left(E_{e e}\right)
$$

where $E_{i 1}$ and $E_{i 2}$ are the boundaries of bin $i$ and $\Delta E_{i}=E_{i 2}-E_{i 1}$. The observed modulation and rate in bin $i$ are then $A_{i}=\left\langle A_{R}\right\rangle_{i}$ and $R_{i}=\langle\bar{R}\rangle_{i}$, respectively. Now we have to perform the bin average of eq. (B4). The first term in the square bracket gives

$$
\frac{\bar{R}\left(E_{i 1}\right) \xi\left(E_{i 1}\right)-\bar{R}\left(E_{i 2}\right) \xi\left(E_{i 2}\right)}{\Delta E_{i}} \approx \frac{R_{i}\langle\xi\rangle_{i}-R_{i+1}\langle\xi\rangle_{i+1}}{\Delta E_{i}} .
$$


For the other two terms in the square bracket we approximate $\langle X Y\rangle \approx\langle X\rangle\langle Y\rangle$ and the integral becomes a sum over bins, up to the last reported bin $i=N$. Finally we obtain

$$
A_{i} \leq v_{e}\left[R_{i}\left(\alpha_{i}+\beta_{i}\right)-R_{i+1} \alpha_{i+1}^{\prime}-\langle\kappa\rangle_{i} \sum_{j=i}^{N} R_{j} \gamma_{j}\right]
$$

with

$$
\alpha_{i} \equiv \frac{\langle\xi\rangle_{i}}{\Delta E_{i}}, \alpha_{i}^{\prime} \equiv \frac{\langle\xi\rangle_{i}}{\Delta E_{i-1}}, \beta_{i} \equiv\left\langle\frac{d \xi}{d E_{e e}}+\sqrt{\frac{2 \mu_{\chi A}^{2} Q}{m_{A} E_{e e}}}+\frac{\xi}{\kappa} \frac{d \kappa}{d E_{e e}}\right\rangle_{i}, \gamma_{i} \equiv \Delta E_{i}\left\langle\frac{2 \mu_{\chi A}^{2} Q}{m_{A} E_{e e} \xi \kappa}\right\rangle_{i} .
$$

For eq. (B5) we get

$$
A_{i} \leq v_{e} \sin \alpha_{\text {halo }}\left[R_{i}\left(\alpha_{i}+\beta_{i}^{\prime}\right)-R_{i+1} \alpha_{i+1}^{\prime}\right] \quad \text { with } \quad \beta_{i}^{\prime} \equiv\left\langle\frac{d \xi}{d E_{e e}}+\frac{\xi}{\kappa} \frac{d \kappa}{d E_{e e}}\right\rangle_{i}
$$

Terms with indices larger than $N$ are dropped. We see from the definitions that $\alpha_{i}, \alpha_{i}^{\prime}, \gamma_{i}$, and $\langle\kappa\rangle_{i}$ are positive.

A similar calculation for the bounds (30) and (31), following from the Assumptions 2 and $2 \mathrm{a}$, leads to

$$
\sum_{j=i}^{N} A_{j} x_{j} \leq v_{e} \sin \alpha\left[R_{i} y_{i}-\left\langle v_{m}\right\rangle_{i} \sum_{j=i+1}^{N} R_{j} \gamma_{j}\right]
$$

with

$$
x_{i} \equiv \Delta E_{i}\left\langle\frac{1}{\xi \kappa}\right\rangle_{i}, \quad y_{i} \equiv\left\langle\frac{1}{\kappa}\right\rangle_{i}-\left\langle v_{m}\right\rangle_{i} \gamma_{i}, \quad\left\langle v_{m}\right\rangle_{i}=\left\langle\sqrt{\frac{m_{A} E_{e e}}{2 \mu_{\chi A}^{2} Q}}\right\rangle_{i},
$$

and $\sin \alpha=1(0.5)$ corresponds to Assumption 2 (2a).

Any binning procedure will lead to inaccuracies. We have estimated binning effects by slightly changing the prescription. Eq. (B4) can be equivalently written as

$$
A_{R}\left(E_{e e}\right) \leq v_{e}\left[-\xi \frac{d \bar{R}}{d E_{e e}}+\bar{R}\left(\sqrt{\frac{2 \mu_{\chi A}^{2} Q}{m_{A} E_{e e}}}+\frac{\xi}{\kappa} \frac{d \kappa}{d E_{e e}}\right)-\kappa \int_{E_{e e}} d E_{e e} \frac{\bar{R}}{\xi \kappa} \frac{2 \mu_{\chi A}^{2} Q}{m_{A} E_{e e}}\right]
$$

The first term in the square bracket can be estimated as

$$
-\xi \frac{d \bar{R}}{d E_{e e}} \approx\langle\xi\rangle_{i} \frac{R_{i}-R_{i+1}}{\Delta E_{i}}
$$

and the other terms are bin-averaged similar as above. We have calculated the CoGeNT bounds also using this binning procedure and obtain similar results as with our default method. This confirms that inaccuracies due to binning are acceptable for present CoGeNT data. 


\begin{tabular}{|c|c|c|c|c|c|c|}
\hline & \multicolumn{3}{|c|}{ unmod. rate from [4] } & \multicolumn{3}{|c|}{ corrected rate $[21]$} \\
\hline & Mean & $68 \%$ & $95 \%$ & Mean & $68 \%$ & $95 \%$ \\
\hline Ass. 1: general bound & 10 & 5 & - & 12.5 & 5 & - \\
\hline Ass. 2: symmetric halo & 29.5 & 17 & 7 & 63 & 34.5 & 16 \\
\hline Ass. 2a: sym. halo, $\sin \alpha=0.5$ & 37.5 & 14 & - & 94.5 & 30.5 & - \\
\hline
\end{tabular}

TABLE III: Lower bounds on the DM mass in GeV, from the requirement that the CoGeNT modulation amplitude is consistent with the upper bound from the unmodulated rate, according to the Assumptions 1, 2, 2a on the DM distribution obtained as described in appendix C. In the left part of the table we use the published unmodulated event rates from [4], whereas for the right part of the table we adopt the preliminary results on surface events contamination at low energies from [21].

\section{Appendix C: Alternative procedure for optimizing the bound in presence of un- known background}

In sec. 5 we have outlined a method based on a least-square minimization ("procedure 2") to find "optimal" values for the coefficients $\omega_{i}$ parameterizing the background contribution in each bin. Here we provide an alternative method for this task.

Let us consider the inequalities (38) and (39) as a system of equations for the $\omega_{i}$. We observe that they have a triangular structure with respect to $\omega_{i}$ : the inequality for a given index $i$ depends only on $\omega_{j}$ with $j \geq i$, i.e., the inequality for $i=N$ depends only on $\omega_{N}$, for $i=N-1$ it depends on $\omega_{N-1}, \omega_{N}$, and so on. From the fact that $\alpha_{i},\langle\kappa\rangle_{i}, \gamma_{i}$ are positive follows that the bound for a given $i$ is weakest if $\omega_{i}$ is as large as possible and $\omega_{j}$ with $j>i$ are as small as possible. We can implement this requirement in an iterative way: replacing the inequality sign by an equality, we obtain a system of $N$ linear equations in $\omega_{i}$. We can solve this system by starting at $i=N$ and going up step by step to $i=1$, in each step obtaining a value for the corresponding $\omega_{i}$, to be used in the next step. If the solution for $\omega_{i}$ is less than one, it will be the smallest allowed value, and hence the bound for $i-1$ will be weakest. If the solution for $\omega_{i}$ is larger than one the bound is violated by bin $i$ and we have to set the corresponding $\omega_{i}=1$ (the largest physically allowed value). In that way we obtain a set of $\omega_{i}$ corresponding to the most conservative choice of background contributions to the unmodulated rate.

This method treats the inequalities as "hard bounds" and does not take into account the fact that the amplitudes are subject to experimental uncertainties. A conservative way to include uncertainties is to apply this procedure as described above but using instead of the 1.h.s. of (38) and (39) its central value minus $n$ standard deviations. In this way one aims to satisfy the inequalities within the $n \sigma$ interval for each bin. We show the results of such an analysis for CoGeNT data in tab. III. Typically one finds qualitatively similar but slightly stronger bounds than for procedure 1, although if the method is considered at higher CL bounds may become weaker or even disappear, as visible in the table for the columns at $95 \%$ CL. Note, however, that subtracting $n \sigma$ from the mean value for all bins leads to very 
conservative limits.

[1] A. K. Drukier, K. Freese, and D. N. Spergel, Detecting Cold Dark Matter Candidates, Phys. Rev. D33, 3495 (1986).

[2] K. Freese, J. A. Frieman, and A. Gould, Signal Modulation in Cold Dark Matter Detection, Phys. Rev. D37, 3388 (1988).

[3] DAMA, R. Bernabei et al., First results from DAMA/LIBRA and the combined results with DAMA/NaI, Eur. Phys. J. C56, 333 (2008), 0804.2741.

[4] C. E. Aalseth et al., Search for an Annual Modulation in a P-type Point Contact Germanium Dark Matter Detector, (2011), 1106.0650.

[5] M. Kuhlen et al., Dark Matter Direct Detection with Non-Maxwellian Velocity Structure, JCAP 1002, 030 (2010), 0912.2358.

[6] N. Fornengo and S. Scopel, Temporal distortion of annual modulation at low recoil energies, Phys. Lett. B576, 189 (2003), hep-ph/0301132.

[7] A. M. Green, Effect of realistic astrophysical inputs on the phase and shape of the WIMP annual modulation signal, Phys. Rev. D68, 023004 (2003), astro-ph/0304446.

[8] M. Fairbairn and T. Schwetz, Spin-independent elastic WIMP scattering and the DAMA annual modulation signal, JCAP 0901, 037 (2009), 0808.0704.

[9] G. Gelmini and P. Gondolo, WIMP annual modulation with opposite phase in Late-Infall halo models, Phys.Rev. D64, 023504 (2001), hep-ph/0012315.

[10] C. Savage, K. Freese, and P. Gondolo, Annual Modulation of Dark Matter in the Presence of Streams, Phys. Rev. D74, 043531 (2006), astro-ph/0607121.

[11] S. Chang, A. Pierce, and N. Weiner, Using the Energy Spectrum at DAMA/LIBRA to Probe Light Dark Matter, Phys.Rev. D79, 115011 (2009), 0808.0196.

[12] A. Natarajan, C. Savage, and K. Freese, Probing dark matter streams with CoGeNT, (2011), 1109.0014.

[13] M. Drees and C.-L. Shan, Reconstructing the Velocity Distribution of WIMPs from Direct Dark Matter Detection Data, JCAP 0706, 011 (2007), astro-ph/0703651.

[14] M. Drees and C.-L. Shan, Model-Independent Determination of the WIMP Mass from Direct Dark Matter Detection Data, JCAP 0806, 012 (2008), 0803.4477.

[15] P. J. Fox, J. Liu, and N. Weiner, Integrating Out Astrophysical Uncertainties, Phys.Rev. D83, 103514 (2011), 1011.1915.

[16] P. J. Fox, G. D. Kribs, and T. M. Tait, Interpreting Dark Matter Direct Detection Independently of the Local Velocity and Density Distribution, Phys.Rev. D83, 034007 (2011), 1011.1910.

[17] C. McCabe, DAMA and CoGeNT without astrophysical uncertainties, (2011), 1107.0741.

[18] M. T. Frandsen, F. Kahlhoefer, C. McCabe, S. Sarkar, and K. Schmidt-Hoberg, Resolving astrophysical uncertainties in dark matter direct detection, (2011), 1111.0292.

[19] J. Read, L. Mayer, A. Brooks, F. Governato, and G. Lake, A dark matter disc in three cosmological simulations of Milky Way mass galaxies, (2009), 0902.0009.

[20] P. J. Fox, J. Kopp, M. Lisanti, and N. Weiner, A CoGeNT Modulation Analysis, (2011), 1107.0717. 
[21] J. Collar, CoGeNT and COUPP, talk at TAUP 2011, "12th International Conference on Topics in Astroparticle and Underground Physics", 2011.

[22] M. T. Frandsen et al., On the DAMA and CoGeNT Modulations, Phys.Rev. D84, 041301 (2011), 1105.3734.

[23] T. Schwetz and J. Zupan, Dark Matter attempts for CoGeNT and DAMA, JCAP 1108, 008 (2011), 1106.6241.

[24] S. Chang, J. Pradler, and I. Yavin, Statistical Tests of Noise and Harmony in Dark Matter Modulation Signals, (2011), 1111.4222.

[25] C. Arina, J. Hamann, R. Trotta, and Y. Y. Wong, Evidence for dark matter modulation in CoGeNT, (2011), 1111.3238.

[26] P. S. Barbeau, J. I. Collar, and O. Tench, Large-Mass Ultra-Low Noise Germanium Detectors: Performance and Applications in Neutrino and Astroparticle Physics, JCAP 0709, 009 (2007), nucl-ex/0701012.

[27] C. McCabe, The Astrophysical Uncertainties Of Dark Matter Direct Detection Experiments, Phys. Rev. D82, 023530 (2010), 1005.0579. 